\title{
Properties of Mass-Loading Shocks 1. Hydrodynamic Considerations
}

\author{
G. P. ZANK AND S. OUghton
}

\author{
Bartol Research Institute, University of Delaware, Newark
}

\begin{abstract}
The one-dimensional hydrodynamics of flows subjected to mass loading are considered anew, with particular emphasis placed on determining the properties of mass-loading shocks. This work has been motivated by recent observations of the outbound Halley bow shock (Neubauer et al., 1990), which cannot be understood in terms of simple hydrodynamical or magnetohydrodynamical descriptions. By including mass injection at the shock, we have investigated the properties of the Rankine-Hugoniot conditions on the basis of a geometric formulation of the entropy condition. Such a condition, which is more powerful than the usual thermodynamical formulation, serves to determine those solutions to the Rankine-Hugoniot conditions which correspond to a physically realizable downstream state. On this basis a concise theoretical description of hydrodynamic mass-loading shocks is obtained. We show that mass-loading shocks have more in common with combustion shocks than with ordinary nonreacting gas dynamical shocks. It is shown that for decelerated solutions to the Rankine-Hugoniot conditions to exist, the upstream flow speed $u_{0}$ must satisfy $u_{0}>u_{\text {crit }}>c_{s}$, where $c_{s}$ is the sound speed. Besides the ùsual supersonic-subsonic transition, mass-loading fronts can also admit a decelerating supersonic-supersonic transition, the structure of which consists of a sharp decrease in the flow velocity preceding a recovery and an increase in the final downstream flow speed. We suggest the possibility that such structures may describe the inbound Halley bow shock (Coates et al., 1987a). Both parallel and oblique shocks are considered, the primary difference being that oblique shocks are subjected to a shearing stress due to mass loading. It is conjectured that such a shearing may destabilize the shock.
\end{abstract}

\section{INTRODUCTION}

Ever since the pioneering work of Axford [1964]; Biermann et al. [1967] (hereinafter referred to as BBS), and Wallis [1971, 1973], research into the interaction of the solar wind with a comet has tended to emphasize the large-scale global dynamics of the flow. Although the bulk of this and subsequent work was given over to the debate concerning the existence or nonexistence of a cometary bow shock, little attention was focused on investigating the properties of shocks dominated by mass loading. This may have been simply a consequence of the uncertainty surrounding the existence of such a structure. BBS used a hydrodynamical description in the presence of a cometary "fluid source" to argue for the existence of a strong bow shock. Wallis [1971] argued, however, that general mass loading of the solar wind may instead allow for the existence of a smooth supersonicsubsonic transition (a "bow wave") or, at least, a shock much weaker than that advocated by BBS.

With the recent flyby missions to comets Giacobini-Zinner (GZ) in 1985 and Halley in 1986 it was hoped that the existence (or nonexistence) of a cometary bow shock would at last be confirmed and some of its properties determined. Unfortunately, the International Cometary Explorer measurements at GZ were not particularly conclusive [Bame et al. 1986], although Smith et al. [1986] did conclude eventually that a weak shock existed. On the other hand, observations made during the Halley encounter provide much clearer evidence for the existence of a shock [Neubauer et al., 1986; Galeev et al., 1986; Mukai et al., 1986; Neugebauer et al., 1987; Coates et al., 1987a, b].

The cometary bow shock observations provide an exciting

Copyright 1991 by the American Geophysical Union.

Paper number 91JA00616.

0148-0227/91/91JA-00616\$05.00 opportunity to investigate in detail the structure and properties of shocks experiencing significant mass loading. Such a study can be initiated at various levels of sophistication and approximation, ranging from the one-fluid to the multifluid [e.g., Sauer, 1988; Zank, 1990] and kinetic descriptions [Omidi and Winske, 1987]. In this and subsequent papers (G. P. Zank and S. Oughton, manuscript in preparation, 1991; G. P. Zank et al., Mass-loading and parallel magnetized shocks, submitted to Geophysical Research Letters, 1991), we use the simple one-fluid model introduced originally by BBS extended to MHD. Our main concern in these papers is to elucidate the properties of mass-loading shocks and to distinguish these properties from those of classical shock theory. It is not widely recognized that with the inclusion of a mass-loading source term in the continuity equation, the nature of the gas dynamic equations is changed from convex to nonconvex (for which definitions are given later). This makes the equations of gas dynamics with mass loading extremely interesting, especially as regards determining the conditions under which shocks can exist without violating the "entropy" condition. In this respect, massloading gas dynamics resembles more closely the fluid dynamics of combustion [e.g., Landau and Lifshitz, 1979] than of ordinary nonreactive gas dynamics.

Our motivation for this work originated with the surprising Giotto observations data obtained on the outbound leg of the Halley encounter presented by Neubauer et al. [1990] and Coates et al. [1990]. It was found that the transverse magnetic field in the shock plane possessed the characteristics of a switch-on shock even though the plasma beta $\beta_{p}$ (the ratio of the total plasma pressure to the magnetic pressure) was very high (in excess of 5). This is in marked contrast to classical MHD shock theory in which the existence of a switch-on shock is permitted only when $\beta_{p}<2 / \gamma$, where $\gamma$ is the adiabatic index of a perfect gas. Neubauer et 
al. [1990] argued that the strong rotation of the magnetic field could be understood on the basis of mass-loading modified Rankine-Hugoniot (R-H) conditions. Unfortunately, their paper was more observationally oriented, and no detailed analysis of this problem was undertaken. An immediate and obvious question that arises concerns the existence of shocks in which the magnetic field is strongly rotated: a strongly rotated downstream magnetic field implies that the downstream gas pressure need not be as large as that for classical MHD shocks (in fact, if the magnetic field is sufficiently strongly rotated, solutions to the mass-loading modified R-H conditions can be found for which the downstream gas pressure is less than that upstream of the shock; that is, the shock is noncompressive (G. P. Zank et al., 1991)). Can these shocks possibly exist, and how does one decide on the admissibility of a solution to the modified $\mathrm{R}-\mathrm{H}$ conditions? In this paper we describe an approach to decide on such issues and apply these methods to the simpler hydrodynamic problem. We defer for subsequent papers the question of MHD. Even for the hydrodynamical problem, there exist significant differences between nonreactive gas dynamical and mass-loading shocks. Thus it is crucial that care be exercised in applying results obtained from classical gas dynamics to mass-loading structures such as cometary bow shocks.

This paper is organized as follows. In section 2 a mathematical treatment of the theory of shocks is presented in a way that seems plausible, but proofs are omitted. A simplified form of the one-dimensional gas dynamics equations with mass loading is presented in section 3 , and an analysis for parallel and oblique planar shocks is carried out in sections 3.1 and 3.2. The results and implications are discussed briefly in section 4 .

\section{Preliminaries}

The second law of thermodynamics asserts that an "entropy" $S$ per unit volume exists for which the property $d S / d t \geq 0$ holds; that is, $S$ increases when energy is converted from kinetic to internal energy. For ordinary gas dynamics it can be shown [e.g., Landau and Lifshitz, 1979] that only compressive shocks are thermodynamically admissible. Compressible shocks are equivalent, in gas dynamics, to shocks which undergo a supersonic-subsonic transition. For complicated reacting flows, such as combustible flows or mass-loading flows, where the thermodynamic properties are either extremely complicated or only partially understood, the physical notion of entropy is unlikely to be of much help in determining which solutions of the R-H conditions are physically sensible. Fortunately, however, the subject of weak or distribution solutions to hyperbolic systems of equations is a topic of considerable interest in the mathematical community, and a rigorous mathematical theory for systems of conservation laws has been developed [Lax, 1973].

Consider the simple scalar conservation law

$$
u_{t}+f(u)_{x}=0
$$

with initial data

$$
\begin{array}{ll}
u(x, 0)=u_{r} & \text { if } x \geq 0 \\
u(x, 0)=u_{l} & \text { if } x<0,
\end{array}
$$

where it is assumed that $f(u)$ is a genuinely nonlinear function (i.e., $f^{\prime \prime}(u) \neq 0$, thereby implying that $f$ is convex). Typically, the problem (1), (2) has a continuum of solutions, so some principle is required to isolate the "physically relevant" solution. Equation (1) can be rewritten as

$$
u_{t}+f^{\prime}(u) u_{x}=0
$$

indicating that $u$ is constant along the characteristics

$$
d x / d t=f^{\prime}(u) \text {. }
$$

A piecewise continuous solution is a solution in the distribution sense if the Rankine-Hugoniot relation

$$
s[u]=[f(u)]
$$

is satisfied across the discontinuity, where brackets denote the difference across the discontinuity, and $s$ is the velocity at which the discontinuity propagates. Clearly, if the piecewise continuous distribution solution is to be determined uniquely, then it is necessary that the characteristics on either side of the discontinuity impinge in the forward $t$ direction on the discontinuity (see, for example, John [1982, p. 16]). Given that the signal velocities on the left and right sides of the discontinuity are $f^{\prime}\left(u_{l}\right)$ and $f^{\prime}\left(u_{r}\right)$ (equation (3)), we can express this condition in terms of the inequality

$$
f^{\prime}\left(u_{l}\right)>s>f^{\prime}\left(u_{r}\right),
$$

where, from (5),

$$
s=\frac{f\left(u_{l}\right)-f\left(u_{r}\right)}{u_{l}-u_{r}}=f^{\prime}(\xi) .
$$

The entropy inequality (6) states that the shock speed is intermediate to the characteristics on both sides of the shock, and for the simple scalar conservation law (1) this additional criterion is sufficient to ensure that the initial value problem is determined uniquely. A graphical depiction of the entropy condition for $f=u^{2} / 2$ appropriate to the two sets of initial data

$$
\begin{array}{ll}
u(x, 0)=0 & \text { if } x \geq 0 \\
u(x, 0)=1 & \text { if } x<0
\end{array}
$$

and

$$
\begin{array}{ll}
u(x, 0)=1 & \text { if } x \geq 0 \\
u(x, 0)=0 & \text { if } x<0
\end{array}
$$

is to be found in Figure 1.

Consider now the linear scalar equation

$$
u_{t}+a u_{x}=0, \quad x>0, \quad t>0,
$$

in the quarter plane, $a \equiv$ const. Suppose that we are given the initial conditions $u(x, 0)$ in $x \geq 0$ and the boundary conditions $u(0, t)$ in $t \geq 0$. Can these initial values determine $u$ in the entire quarter plane? Since $u$ is constant along the lines $x-a t=$ const, if $a<0$, then $u$ is determined along $x=0$ by its initial values (see Figure $2 a$ ) and no boundary conditions can therefore be given. Conversely, for $a>0$, it is clear from Figure $2 b$ that the boundary conditions along $\boldsymbol{x}=\mathbf{0}$ must be given in order to determine $u$ in the full quarter plane. 


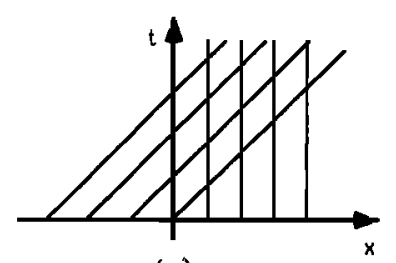

(a)

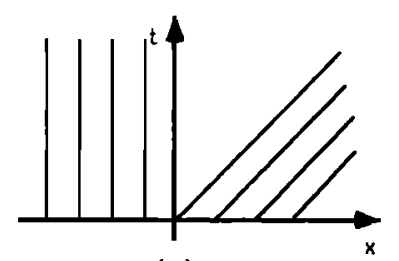

(c)

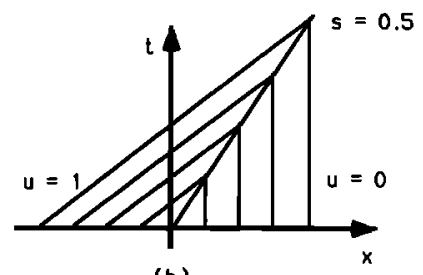

(b)

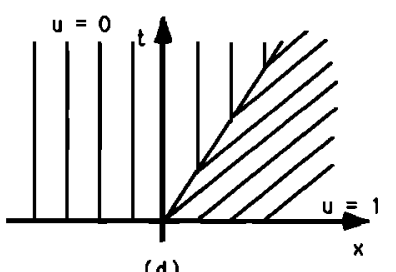

(d)

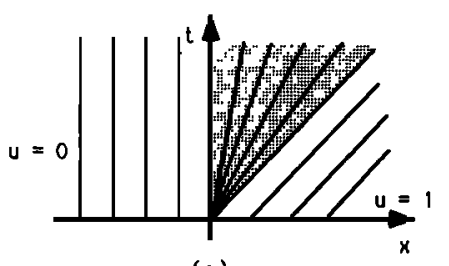

(e)

Fig. 1. Examples of the conservation law $u_{t}+\left(u^{2} / 2\right)_{x}=0$ with the two sets of initial data $u(x, 0)=0$ if $x \geq 0$ and $u(x, 0)=1$ if $x<0$ (Figures $1 a$ and $1 b$ ) and $u(x, 0)=1$ if $x \geq 0$ and $u(x, 0)=$ 0 if $x<0$ (Figures $1 c-1 e$ ). To prevent the characteristics in Figure $1 a$ from crossing, a shock must be introduced with propagation speed $s=\left[u^{2} / 2\right] /[u]=1 / 2$. Thus a globally defined weak solution is given by $u=1$ on the left of the shock and $u=0$ on the right. For the second set of initial data, the characteristics do not fill out the ( $x$, $t$ ) plane (Figure 1c). Two approaches to defining a globally valid weak solution are illustrated in Figures $1 d$ and $1 e$. A criterion is given in the text which excludes solutions of the kind illustrated in Figure $1 d$. The solution exhibited in Figure $1 e$ corresponds to a rarefaction fan (the shaded region) and is often utilized in the construction of compound wave fronts.

The extension of these ideas to a hyperbolic system of $n$ equations is readily accomplished. Suppose that instead of the quarter-plane problem above, we have a discontinuity which moves with speed $s(s=0$ for the quarter-plane problem) and let $\lambda_{1}(u)<\cdots<\lambda_{n}(u)$ denote the eigenvalues, i.e., the characteristic speeds of the system (e.g., for one-dimensional gas dynamics, the three eigenvalues are, of

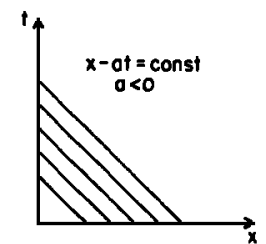

(o)

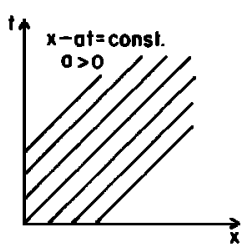

(b)

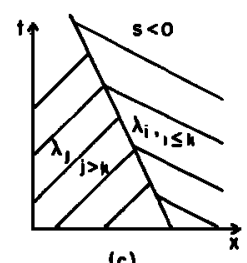

(c)

Fig. 2. Graphical determination of the boundary conditions appropriate to a stationary shock. See text for details. course, $u \pm c, u)$. Let $u_{l}, u_{r}$ denote the state vectors on either side of the discontinuity and suppose that $\lambda_{1}\left(u_{r}\right)$ $<\cdots<\lambda_{k}\left(u_{r}\right)<s<\lambda_{k+1}\left(u_{r}\right)<\cdots<\lambda_{n}\left(u_{r}\right)$. Extending the reasoning used for the scalar equation, but now for the region $(x-s t)>0, t>0$, if $i \leq k$, then since $\lambda_{i}\left(u_{r}\right)$ $<s$, the $u^{i}(0, t)$ are determined by the initial data. If $i>k$, then $\lambda_{i}\left(u_{r}\right)>s$ and we must specify $u^{t}(0, t), i=k+$ $1, \cdots, n$. Thus we should specify $(n-k)$ conditions on the right boundary of the discontinuity. Similarly, for the left boundary, if $\lambda_{j}\left(u_{l}\right)<s<\lambda_{j+1}\left(u_{l}\right)$, we must specify $j$ conditions on the left boundary of the discontinuity (Figure 2c). The jump conditions

$$
s[u]=[f(u)]
$$

provide $n$ algebraic equations connecting the values on both sides of the discontinuity with $s$. But since $u_{l} \neq u_{r}$, this reduces to $(n-1)$ conditions between $u_{l}$ and $u_{r}$. Thus it is required that

$$
(n-k)+j=n-1
$$

or

$$
j=k-1 \text {. }
$$

In the light of this discussion we should admit a discontinuity $\left(u_{l}, u_{r} ; s\right)$ provided that for some index $k, 1 \leq k \leq n$, the following inequalities hold:

$$
\begin{aligned}
& \lambda_{k}\left(u_{r}\right)<s<\lambda_{k+1}\left(u_{r}\right) \\
& \lambda_{k-1}\left(u_{l}\right)<s<\lambda_{k}\left(u_{l}\right) .
\end{aligned}
$$

These inequalities define an entropy condition (sometimes called the Lax inequalities and here called the geometrical entropy condition), and any discontinuity satisfying (10) is called a shock (sometimes a $k$ shock). The entropy condition can also obviously be written in the equivalent form

$$
\begin{gathered}
\lambda_{k}\left(u_{r}\right)<s<\lambda_{k}\left(u_{l}\right) ; \\
\lambda_{k-1}\left(u_{l}\right)<s<\lambda_{k+1}\left(u_{r}\right),
\end{gathered}
$$

which is often an easier form to use. Thus for only one index $k$ is the shock speed $s$ intermediate to the characteristic speeds $\lambda_{k}$ on both sides of the shock. A discussion relating (9) and (10) to the stability of shocks is given by Liberman and Velikovich [1986].

Lax [1973] has shown that for an ideal gas a shock is compressive if and only if it satisfies the entropy condition (10). Thus for ideal gases the geometric entropy condition and the "physical" entropy condition obtained from thermodynamic arguments happen to coincide. This need not always be the case. The geometric entropy condition is stronger and may be needed for the construction of a unique solution, even under circumstances where thermodynamics has little to say.

The utility of the entropy condition (11) lies in its simple graphical representation in the $(x, t)$ plane. At this stage, some definitions are in order. A family of characteristics is said to cross a discontinuity that satisfies the $\mathbf{R}-\mathbf{H}$ conditions if through every point of the discontinuity in the $(x, t)$ plane one can draw only one characteristic of that family, with the property that it is traceable backward in time on one side of the discontinuity and forward on the other. This corresponds 
to a family of characteristics crossing a discontinuity that is not required to prevent intersection of the characteristics. A family of characteristics is linearly degenerate if a discontinuity that satisfies the $\mathrm{R}-\mathrm{H}$ conditions happens to coincide with a member of that family. The $C_{0}$ characteristic of gas dynamics is an example. A discontinuity separates a family of characteristics if through every point of the shock trajectory in the $(x, t)$ plane, there exists a pair of characteristics which can be traced either backward or forward in time. The entropy condition (11) can therefore be reformulated as follows: A discontinuity satisfies the entropy condition if, when it separates the characteristics of a family, the characteristics on each side can be traced back to the initial data. A family of characteristics is convex if a discontinuity satisfying the $\mathrm{R}-\mathrm{H}$ conditions is either crossed by the family or separates the family. The $C_{+}$and $C_{-}$characteristics of ideal gas dynamics are examples of convex families.

A system of conservation laws is classified as convex if all families of characteristics are convex and if all the discontinuities admitted by the $\mathrm{R}-\mathrm{H}$ conditions separate one and only one of the families. The isentropic gas dynamics equations are convex. If, however, at least one of the families is linearly degenerate and the others convex, then the system of conservation laws is linearly degenerate. The ideal gas dynamics equations represent the standard example. For any other possibilities the system is described as nonconvex, and compound waves are often needed to connect states and certainly to construct a solution of the Riemann problem. The most studied example of a nonconvex system is that of gas dynamics with combustion.

\section{Mass-Loading Shocks}

As a comet approaches the Sun, neutral molecules and dust are liberated from the nucleus with a typical speed of 1 $\mathrm{km} \mathrm{s}^{-1}$. After some $10^{6} \mathrm{~s}$ the particles are ionized, whereupon they begin to gyrate about the interplanetary magnetic field. Since the gyration begins almost instantaneously on ionization, a common velocity of the plasma components is established (within the plane of gyration) very rapidly via pitch angle scattering [Neugebauer et al., 1987]. Thus besides validating the assumption of a common bulk velocity for all plasma components, it also suggests that one should choose the ratio of specific heats $\gamma$ to be $5 / 3$, appropriate to 3 degrees of freedom, rather than the commonly used $\gamma=2$ (BBS) which takes into account only 2 degrees of freedom. In principle, because the ions cannot completely share their energy with the electrons, we assume that the electron component is cold and does not influence the large-scale flow. Finally, since the newly ionized particles have a very small initial velocity, they add negligibly to the overall energy and momentum balance so we need account for their presence only in the total mass flux equation. This assumption is relaxed by G. P. Zank et al. (submitted manuscript, 1991), who show explicitly that the additional terms are unimportant. Thus, as recognized by Axford [1964] and BBS, it is the addition of mass that tends to dominate the solar wind-comet interaction. However, if one works in a frame of reference different from the cometary reference frame, momentum and energy source terms may well need to be retained under certain circumstances (see Neubauer et al. [1990] and Section 3.2).

Two important points for our analysis emerge from the observations presented by Neubauer et al. [1990] (data obtained on the outbound leg of the Giotto mission). The first is that the thickness of the "outbound" shock in the normal direction is $d=120,000 \mathrm{~km}$, which implies that the shock is still relatively thin when compared to typical length scales in the system. Thus we may assume a one-dimensional model when investigating properties of the shock. Second, using the gas production rates of Krankowsky et al. [1986] and a $10^{6} \mathrm{~s}$ lifetime for the dissociation products of cometary neutral molecules gives an injected mass-loading mass flux of the order of $1.4 \times 10^{7} \mathrm{amu} / \mathrm{cm}^{2} \mathrm{~s}$. On the other hand, Coates et al. [1990] have measured the upstream mass flux to be $2.3 \times 10^{8} \mathrm{amu} / \mathrm{cm}^{2} \mathrm{~s}$, giving a significant mass loading to upstream mass flux ratio of $\sim \mathbf{0 . 0 1}$. For the inbound Halley shock the measured shock thickness was found to be $\sim 40,000-45,000 \mathrm{~km}$ instead [Coates et al., 1987a, b], which yields a mass loading to upstream mass flux ratio of $\sim 0.005$.

On the basis of the observations above, we can write down an idealized mathematical model describing mass loading in a flow. We assume that at some location in the shock frame of the flow, new particles are injected continuously into the fluid in such a way that mass is added but not energy or momentum. The one-dimensional idealized gas dynamics equations with mass loading can then be written as

$$
\begin{gathered}
\frac{\partial \rho}{\partial t}+\frac{\partial}{\partial x}\left(\rho u_{x}\right)=\alpha \delta(x-s t) \\
\frac{\partial}{\partial t}\left(\rho u_{x}\right)+\frac{\partial}{\partial x}\left(\rho u_{x}^{2}+p\right)=0 \\
\frac{\partial}{\partial t}\left(\rho u_{y}\right)+\frac{\partial}{\partial x}\left(\rho u_{x} u_{y}\right)=0 \\
\frac{\partial e}{\partial t}+\frac{\partial}{\partial x}\left((e+p) u_{x}\right)=0
\end{gathered}
$$

where $e \equiv \rho u^{2} / 2+\rho \varepsilon$ and $\varepsilon$ is the internal energy of the fluid. Here $\rho$ denotes the fluid density, $\mathbf{u}=\left(u_{x}, u_{y}\right)$ the velocity field, $p$ the gas pressure, and $\alpha \equiv q m_{c} d$ the "averaged" source term ( $q \equiv$ average production rate of cometary ions, $m_{c}$ is the mass of a cometary ion, and $d$ is the shock thickness) and, finally, $s$ represents the speed of propagation of the shock. For an ideal gas (the case we consider),

$$
\varepsilon=\frac{1}{\gamma-1} \frac{p}{\rho}
$$

so that

$$
e+p=\frac{1}{2} \rho u^{2}+\frac{\gamma}{\gamma-1} p .
$$

The shock configuration is illustrated in Figure 3 for a left-facing shock propagating with velocity $s$.

Some comments regarding our mathematical formulation (12)-(15) are in order. It should be recognized that in a "real" mass-loading system, such as occurs at comets, Venus, Mars, etc., mass loading is omnipresent over very extensive scales. Thus the upstream supersonic unshocked flow is already decelerated as a consequence of mass loading, and the flow velocity is therefore nonconstant ahead of the shock. To determine the location of the subshock, it is 


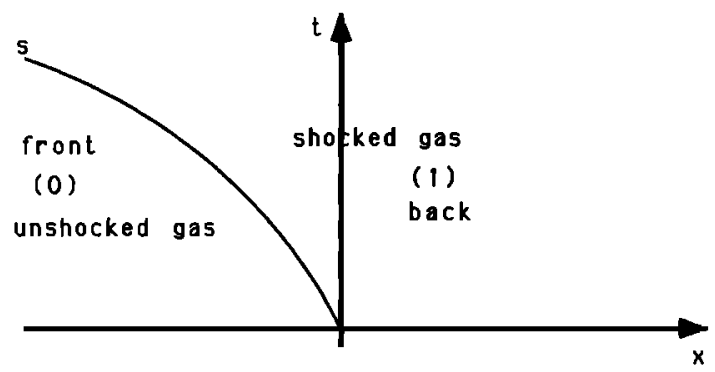

Fig. 3. Schematic of a left-facing shock propagating with speed $s$ in the $(x, t)$ plane.

necessary to consider the full global problem, which includes mass loading far upstream [e.g., BBS; Wallis, 1971, 1973]. To fit a gas subshock in order to affect a transition to the final downstream state, one needs to know only the state of the fluid immediately ahead of the shock and thereafter to apply the usual gas dynamic Rankine-Hugoniot conditions. A very clear exposition of this procedure as applied to cosmic ray mediated shocks is given by Axford et al. [1982]. Thus a typical calculation will neglect mass loading within the subshock and use the standard gas dynamical shock analysis to determine properties of the (sub)shock [e.g., Coates et al., 1987a, b, 1990]. As indicated in section 1, our intention is not to perform a global analysis of mass-loading flows (and thereby establish both the necessity and possible location of the subshock) but rather to investigate in detail the nature of the subshock when mass loading is assumed to occur within the transition. As we show below, this yields shock properties quite different from those of nonreacting gas dynamics. ive do, however, make the assumption that the mass-loading term $\alpha$ is constant throughout the transition, but since the thickness of the shock is expected to vary as a function of incident gas Mach number $M_{0}$, it is possible that $\alpha=$ $\alpha\left(M_{0}\right)$. We do not address this additional complication here. The other important assumption implicit in our analysis is that the actual thickness of the subshock transition is much less than the length scale of deceleration induced by mass loading in the foreshock region. This is equivalent to assuming that no steep gradients are present in the foreshock region, an assumption which is supported observationally at Halley but perhaps less clearly at comet GZ. We draw attention to one final point concerning the downstream state of the shocked fluid. Since, in reality, mass loading of the solar wind continues downstream of the shock, the shocked fluid will obviously continue to evolve in response to the additional cometary ion injection. Therefore the calculated downstream states admitted by the subshock are most likely valid for only a short distance beyond the location of the sharp flow transition.

Provided that $\Psi=(\rho, \mathbf{u}, p)^{t}$ is $C^{1}(\mathscr{R}, t>0)$, the conservation law (15) can be reduced to

$$
\frac{\partial p}{\partial t}+u_{x} \frac{\partial p}{\partial x}+\gamma p \frac{\partial u_{x}}{\partial x}=\frac{\gamma-1}{2} \alpha u^{2} \delta(x-s t),
$$

illustrating that mass loading can be interpreted as a "dissipative process." Thus, for $\Psi$ smooth, equations (12)-(15) can be expressed as the hyperbolic system

$$
\frac{\partial \Psi}{\partial t}+A(\Psi) \frac{\partial \Psi}{\partial x}=B(\Psi)
$$

where $A$ and $B$ are defined to be

$$
A(\Psi)=\left(\begin{array}{cccc}
u_{x} & \rho & 0 & 0 \\
0 & u_{x} & 0 & \frac{1}{\rho} \\
0 & 0 & u_{x} & 0 \\
0 & \gamma p & 0 & u_{x}
\end{array}\right)
$$$$
B(\Psi)=\alpha \delta(x-s t)\left(1,-\frac{u_{x}}{\rho},-\frac{u_{y}}{\rho}, \frac{\gamma-1}{2} u^{2}\right)^{\prime},
$$

$t$ indicating transpose. The characteristics of (3.7) are simply those of ordinary gas dynamics

$C_{+}$:

$$
d x / d t=u_{x}+c
$$

$C_{-}$:

$$
d x / d t=u_{x}-c
$$

$C_{0}$ :

$$
d x / d t=u_{x},
$$

where $c=(\gamma p / \rho)^{1 / 2}$ is the sound speed.

In the frame of the shock (i.e., $s=0$ ) we can write the jump conditions as

$$
\begin{gathered}
\rho_{0} u_{x 0}=\rho_{1} u_{x 1}-\alpha ; \\
\rho_{0} u_{x 0}^{2}+p_{0}=\rho_{1} u_{x 1}^{2}+p_{1} ; \\
\rho_{0} u_{x 0} u_{y 0}=\rho_{1} u_{x 1} u_{y 1} ; \\
\left(e_{0}+p_{0}\right) u_{x 0}=\left(e_{1}+p_{1}\right) u_{x 1} .
\end{gathered}
$$

For the present, we regard $\alpha$ as constant. The above integrated conservation laws resemble those given by $\mathrm{Neu}$ bauer et al. [1990].

\subsection{Parallel Shocks}

Most of the points we wish to emphasize can be made by considering the less general case of flows whose velocity is normal to the one-dimensional planar mass-loading front. Thus we consider $u_{y}=0$, and for notational convenience we set $u_{x}=u$ for this subsection. It should be noted, however, that for MHD mass-loading shocks, obliquity determines some of the most important properties. Some simple but useful algebraic identities for a mass-loading hydrodynamic shock can be derived from the R-H relations. Let $m=\rho_{0} u_{0}$ and introduce the specific volume $\tau=1 / \rho$. Then using $u_{0}=$ $m \tau_{0}$ and $u_{1}=m(1+\bar{\alpha}) \tau_{1} \equiv m \bar{\tau}_{1}, \bar{\alpha} \equiv \alpha / m$, in the momentum relation (22) yields

$$
\begin{gathered}
m=\frac{p_{1}-p_{0}}{u_{0}-(1+\bar{\alpha}) u_{1}} \\
m^{2}=\frac{p_{1}-p_{0}}{\tau_{0}-(1+\bar{\alpha}) \bar{\tau}_{1}} .
\end{gathered}
$$

The total energy relation (24) reduces to 


$$
e_{0} \tau_{0}-e_{1} \bar{\tau}_{1}=p_{1} \bar{\tau}_{1}-p_{0} \tau_{0}
$$

We can further show that

$$
e_{0} \tau_{0}-e_{1} \bar{\tau}_{1}=\varepsilon_{0}-\bar{\varepsilon}_{1}+\frac{p_{1}-p_{0}}{2}\left(\tau_{0}+\bar{\tau}_{1}\right)+\frac{\bar{\alpha}}{2} m^{2} \tau_{0} \bar{\tau}_{1},
$$

where $\bar{\varepsilon}=(1+\bar{\alpha}) \varepsilon$. Thus we may introduce the massloading form of the Hugoniot function

$$
\begin{aligned}
H(\bar{\tau}, p) \equiv \bar{\varepsilon}(\bar{\tau}, p) & -\varepsilon\left(\tau_{0}, p_{0}\right) \\
& +\frac{p+p_{0}}{2}\left(\bar{\tau}-\tau_{0}\right)-\frac{\bar{\alpha}}{2} m^{2} \tau_{0} \bar{\tau},
\end{aligned}
$$

and the Hugoniot equation is $H\left(\bar{\tau}_{1}, p_{1}\right)=0$. Expression (26) differs from the standard gas dynamic Hugoniot function both in the term $(1+\bar{\alpha}) \varepsilon$ and in $\bar{\alpha} m^{2} \tau_{0} \bar{\tau} / 2$. For an ideal gas, (26) can be rearranged as

$$
\begin{aligned}
2 \mu^{2} H(\bar{\tau}, p)=\left(\bar{\tau}-\mu^{2} \tau_{0}\right) p-\left(\tau_{0}-\mu^{2} \bar{\tau}\right) p_{0} \\
-\bar{\alpha} \mu^{2} m^{2} \tau_{0} \bar{\tau},
\end{aligned}
$$

and $\mu^{2}=(\gamma-1) /(\gamma+1)$. Note that a state cannot be connected to itself by a mass-loading front since $H\left(\bar{\tau}_{0}, p_{0}\right)$ $\neq 0$. This has important implications for the development of a theorem analogous to that given by Weyl [1949] for ordinary gas dynamics, and this provides a different perspective on the inability of the physical entropy condition to select the physically relevant $\mathrm{R}-\mathrm{H}$ solutions. This is discussed by Zank [1991]. For a given $\bar{\alpha}, H(\bar{\tau}, p)=0$ is the locus of all possible states that can be connected to the given state $\left(\tau_{0}, p_{0}\right)$. Observe also that unlike the usual gas dynamic Hugoniot, the mass-loading form is a function of the upstream Mach number through $m^{2}$. This is evident if one introduces

$$
M^{2}=u^{2} / c^{2}=u^{2} / \gamma p \tau
$$

since then $H(\bar{\tau}, p)=0$ reduces to

$$
\frac{p}{p_{0}}=\frac{1-\mu^{2} \bar{\tau} / \tau_{0}}{\bar{\tau} / \tau_{0}-\mu^{2}}+\bar{\alpha} \mu^{2} \gamma M_{0}^{2} \frac{\bar{\tau} / \tau_{0}}{\bar{\tau} / \tau_{0}-\mu^{2}} .
$$

Thus $p / p_{0}$ is hyperbolic in $\bar{\tau} / \tau_{0}$ with asymptotes

$$
\begin{gathered}
\frac{\tau}{\tau_{0}}=\frac{\mu^{2}}{1+\bar{\alpha}} \\
\frac{p}{p_{0}}=-\mu^{2}\left(1-\bar{\alpha} \gamma M_{0}^{2}\right) .
\end{gathered}
$$

Clearly, depending on how supersonic the upstream flow is, the $\bar{\tau} / \tau_{0} \rightarrow \infty$ asypmtote can lie either in the $p>0$ or the $p<0$ half plane. The initial state $\left(\tau_{0}, p_{0}\right)$ can also lie above or below the Hugoniot, depending on the value of $M_{0}^{2}$. By considering $H\left(\bar{\tau}_{0}, p_{0}\right)$, it is easily seen that if

$$
M_{0}^{2}>\frac{1}{1+\bar{\alpha}} \frac{2}{\gamma-1}
$$

then $\left(\tau_{0}, p_{0}\right)$ lies below the Hugoniot, otherwise $\left(\tau_{0}, p_{0}\right)$ is above the Hugoniot curve.
Solutions to the R-H conditions can be obtained by solving (28) simultaneously with the total momentum equation

$$
\frac{p}{p_{0}}=\left(1+\gamma M_{0}^{2}\right)-\gamma M_{0}^{2}(1+\bar{\alpha}) \frac{\bar{\tau}}{\tau_{0}}
$$

now written in terms of the upstream Mach number. The R-H conditions are solved graphically for three different cases in Figure 4. In Figures $4 a$ and $4 c,\left(\tau_{0}, p_{0}\right)$ lies below the Hugoniot by virtue of our choices of $\bar{\alpha}$ and $M_{0}^{2}$. Not all points on the Hugoniot correspond to physically admissible downstream states. On rewriting expression (25) in terms of the upstream Mach number,

$$
\gamma M_{0}^{2}=\frac{1}{(1+\bar{\alpha})^{2}} \frac{p / p_{0}-1}{1 /(1+\bar{\alpha})^{2}-\tau / \tau_{0}},
$$

it is evident that in the quarter plane defined by

$$
\begin{array}{rlrl}
p / p_{0}>1 ; & p / p_{0} & <1 \\
\tau / \tau_{0}>\frac{1}{(1+\bar{\alpha})^{2}} ; & \tau / \tau_{0}<\frac{1}{(1+\bar{\alpha})^{2}},
\end{array}
$$

we have $M_{0}^{2}<0$, which is clearly an invalid solution of the R-H conditions. On the Hugoniot, $M_{0}^{2}<0$ corresponds to the segment marked $m^{2}<0$ in Figure $4 a$, so we reject any downstream states corresponding to this region.

To determine which sections of the Hugoniot correspond to subsonic or supersonic downstream flows, we have included the sonic or acoustic line $u_{1}^{2}=c_{1}^{2}$ in Figures 4. The downstream sound speed relation assumes the form

$$
\frac{p}{p_{0}}=(1+\bar{\alpha})^{2} M_{0}^{2} \frac{\tau}{\tau_{0}} .
$$

In Figure $4 a$, where $M_{0}^{2}=10$, we see that two possible downstream states are admitted by the mass-loading R-H conditions, both of which are compressive and whose density increases. Only the most compressive state, however, corresponds to subsonic flows downstream of the shock, whereas the second corresponds to supersonic downstream flows. Conversely, for the subsonic upstream flow example illustrated in Figure $4 b$, two expansive downstream states with decreasing density can be found to solve the R-H conditions. Here, we use the terms expansive/compressive in the sense that either $(1+\bar{\alpha}) \tau_{1}>\tau_{0}$ or $<\tau_{0}$. As in the previous example, one of these states is subsonic and the other supersonic downstream of the discontinuity. Thus we see that mass loading slows and compresses supersonic flows, whereas subsonic flows are accelerated and expanded. This can also be seen if one considers a steady flow, close to equilibrium, and expands the $\mathrm{R}-\mathrm{H}$ conditions appropriately (see the appendix for the general oblique case). The fractional change in velocity after mass loading is then given by

$$
\frac{\delta u}{u_{0}}=\bar{\alpha} \frac{\gamma+1}{2} \frac{M_{0}^{2}}{1-M_{0}^{2}}
$$

Thus if the incident flow is supersonic, i.e., $M_{0}>1$, then $\delta u<0$ indicating deceleration, whereas for flows initially subsonic, $\delta u>0$, illustrating that mass loading can accelerate a subsonic flow. This point was noted already by BBS. 


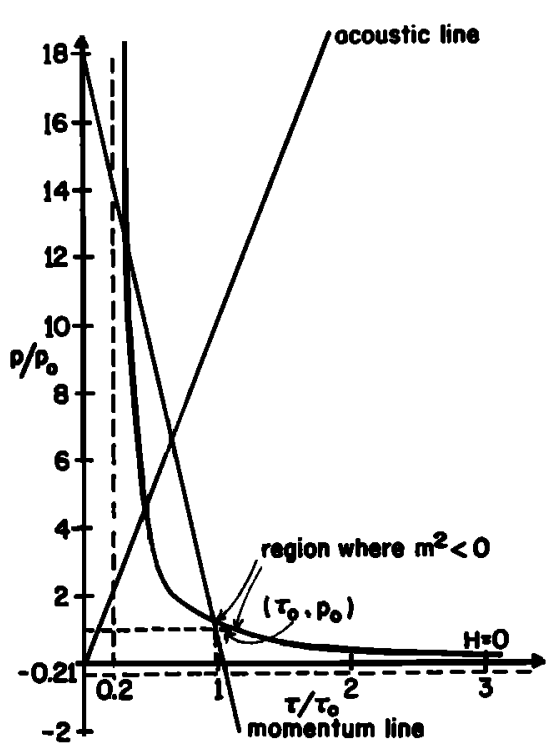

(o)

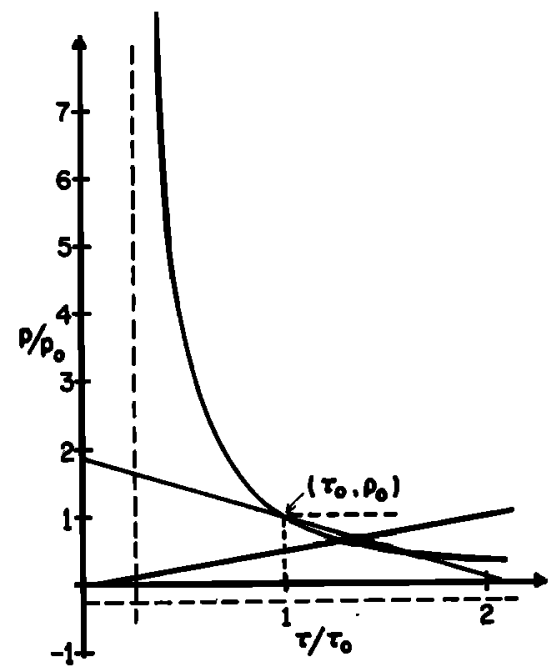

(b)

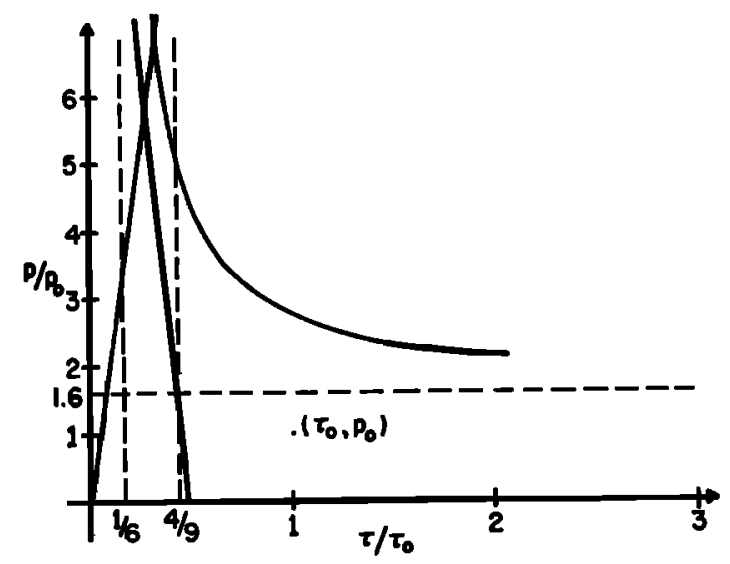

(c)

Fig. 4. Examples of the Hugoniot function together with graphical solutions of the Rankine-Hugoniot conditions, for a parallel shock. $(a) M_{0}^{2}=10$. (b) $M_{0}^{2}=1 / 2, \bar{\alpha}=0.01, \gamma=5 / 3$. (c), $M_{0}^{2}$ $=9$ but $\bar{\alpha}=1 / 2$ and $\gamma=5 / 3$. Possible downstream states correspond to the points of intersection of the total momentum graph and the Hugoniot and the acoustic graph separates subsonic downstream solutions from supersonic. Only for Figure $4 c$ do no downstream states exist.
It is not at all clear, however, that all these flows are dynamically admissible. This has also been emphasized by Wallis [1971, 1973] but from a somewhat different point of view.

To proceed further, we need to classify the Hugoniot more precisely. To this end we can combine equations (28) and (31) to obtain a quadratic equation in $\tilde{\tau} \equiv \bar{\tau} / \tau_{0}$

$$
(1+\bar{\alpha}) \bar{\tau}^{2}-\frac{2 \gamma}{\gamma+1}\left(1+\frac{1}{\gamma M_{0}^{2}}\right) \bar{\tau}+\frac{\gamma-1}{\gamma+1}+\frac{2}{\gamma+1} \frac{1}{M_{0}^{2}}=0
$$

Equation (34) closely resembles an equation of BBS [see Ip and Axford, 1989, equation (2.6)] used to show that a (sub)shock must form in the cometary accretion flow before the condition

$$
\rho u \geq \frac{\gamma^{2}}{\gamma^{2}-1} \rho_{0} u_{0}
$$

is met. It must be emphasized that (34) does not represent a generalization of BBS to flows for which $M_{0}^{2} \neq \infty$. The work of $\mathrm{BBS}$ may be viewed as a shock structure problem in which the source term for mass loading is a prescribed function of $\mathbf{x}$, whereas we are investigating the admissible boundary conditions for a flow in which particles are injected continuously at the shock. Thus unlike BBS we use an averaged, constant source term $\alpha$ so that the solutions of (34) represent possible downstream states which satisfy the $\mathrm{R}-\mathrm{H}$ conditions rather than a condition on the continuously varying mass flux for the insertion of a subshock in the smoothly decelerating flow.

Solutions to (34) correspond to the points of intersection of the Hugoniot and momentum curves in Figure 4 and these can be written explicitly as

$$
\begin{array}{r}
\frac{\tau}{\tau_{0}}=\frac{\gamma}{\gamma+1} \frac{1}{(1+\bar{\alpha})^{2}}\left\{\left(1+\frac{1}{\gamma M_{0}^{2}}\right) \pm\left[\left(1+\frac{1}{\gamma M_{0}^{2}}\right)^{2}\right.\right. \\
\left.\left.-(1+\bar{\alpha}) \frac{\gamma^{2}-1}{\gamma^{2}}\left(1+\frac{2}{\gamma-1} \frac{1}{M_{0}^{2}}\right)\right]^{1 / 2}\right\}
\end{array}
$$

We reiterate that in principle, $\bar{\alpha}=\bar{\alpha}\left(M_{0}\right)$. The momentum line and the Hugoniot are clearly tangential provided $M_{0}^{2}$ satisfies our version of BBS's celebrated condition

$$
(1+\bar{\alpha})=\frac{\gamma^{2}}{\gamma^{2}-1} \frac{\left(1+1 / \gamma M_{0}^{2}\right)^{2}}{1+\frac{2}{\gamma-1} 1 / M_{0}^{2}}
$$

and, obviously, downstream solutions can exist only if $(1+$ $\bar{\alpha}$ ) is less than the right-hand side of (36). Observe that $M_{0}$ can never be 1 . The point at which the momentum line and the Hugoniot are tangent has, however, an added significance. By considering the downstream sound speed relation (32), it is seen that the sonic line and the momentum line intersect at

$$
\frac{\tau}{\tau_{0}}=\frac{\gamma}{\gamma+1} \frac{1}{(1+\bar{\alpha})^{2}}\left(1+\frac{1}{\gamma M_{0}^{2}}\right)
$$




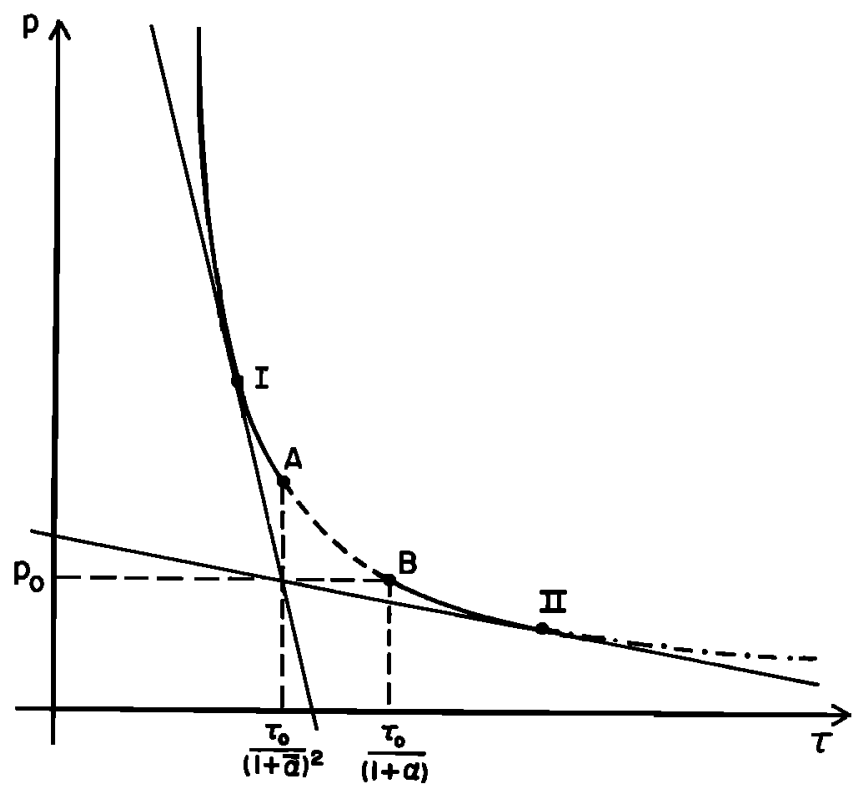

Fig. 5. The classification of the mass-loading Hugoniot. Only the solid lines correspond to possible admissible downstream states. See text for details.

Thus when (36) is satisfied, the point of intersection of the sonic line, the momentum line, and the Hugoniot all coincide. This is sufficient then to provide us with a complete characterization of the mass-loading Hugoniot.

Our results can be summarized in terms of a general representation for the mass-loading Hugoniot (Figure 5). Figure 5 is general in the obvious sense that not all of the marked regions are necessarily present and depends on the location of the two asymptotes (see (29)). In Figure $4 c$, for example, it is evident that the region corresponding to $B$ and downward in Figure 5 is completely absent. Clearly, from (29) Figure 5 is completely general provided $M_{0}^{2}<(1+$ $\left.\mu^{2}\right) /\left(\bar{\alpha} \gamma \mu^{2}\right)$, which, given the parameters quoted above, should hold for mass loading at comets. One portion of the Hugoniot curve, the dashed section from A to $B$, is inadmissible since the states it represents correspond to negative $M_{0}^{2}$. The remaining curve can be separated into two branches, the upper corresponding to a compressive front $\left(p>p_{0}\right.$ ) (in combustion theory, this region would correspond to the detonation branch), whereas the lower portion of the Hugoniot corresponds to an expansive front $\left(p<p_{0}\right)$ (equivalent to the deflagration branch for combustion). The lines through $\left(\tau_{0} /(1+\bar{\alpha})^{2}, p_{0}\right)$ tangent to the Hugoniot at points I and II are the mass-loading versions of the "Rayleigh" lines, and points I and II correspond to the ChapmanJouguet points of combustion theory . Points I and II serve further to divide the Hugoniot curve. The strong compression branch above I corresponds to subsonic flows downstream of the discontinuity. The weak compression regime between I and A corresponds to downstream states with supersonic flows. The branch between B and II describes the weak expansion regime and has subsonic flows downstream of the front. The final branch, the dot-dashed segment from II onward, represents the strong expansion regime with supersonic downstream flows. We will discuss each branch separately and exclude some branches on the basis of the geometric entropy condition discussed in section 2 .

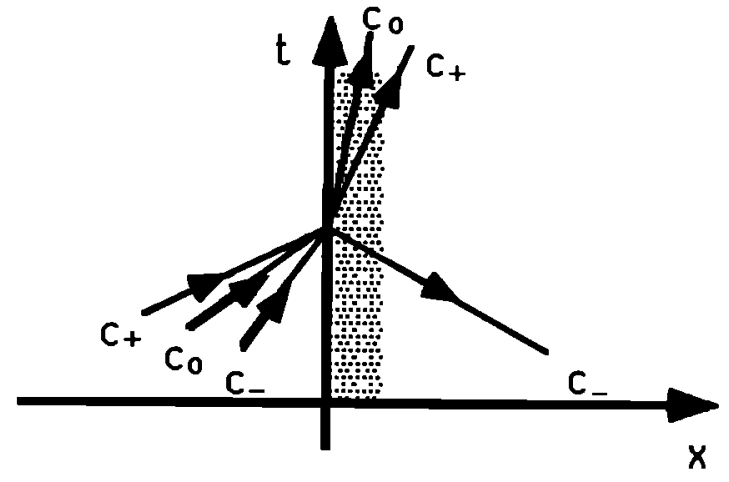

Fig. 6. Geometry of the characteristics corresponding to strong compression branch of the Hugoniot curve.

As a final point, consider the relations (derived below)

$$
\begin{gathered}
u_{0}^{2}-c_{*}^{2}=\left(1-\mu^{2}\right)\left(u_{0}^{2}-c_{0}^{2}\right) \\
u_{0}^{2}-c_{*}^{2}=m^{2} \tau_{0}\left(\tau_{0}-\bar{\tau}_{1}\right)+\bar{\alpha} m^{2} \frac{\tau_{0} \bar{\tau}_{1}^{2}}{\tau_{0}-\bar{\tau}_{1}}
\end{gathered}
$$

where a quantity $c_{*}$ similar to a critical sound speed has been introduced. Equations (37) show that $\left|u_{0}\right|>\left|c_{*}\right| \Leftrightarrow$ $\left|u_{0}\right|>\left|c_{0}\right|$, from which we infer that shock solutions lie on the Hugoniot above the point B (Figure 5) provided $u_{0}>c_{0}$, and below $B$ if $u_{0}<c_{0}$. Thus the strong branch corresponds to supersonic-subsonic transitions, for example.

Let us now consider a constant state $S$ which corresponds to a point $\left(\tau_{1}, p_{1}\right)$ lying on the mass-loading Hugoniot. Then it is possible to connect $S$ and $\left(\tau_{0}, p_{0}\right)$ by means of a mass-loading front. Depending on the location of $S$, we need to consider five cases.

1. For $S$ on the strong compression branch, $p_{1}>p_{I}$. From (35) and (36) it is clear that for solutions to exist on the compressive branch it is necessary that $M_{0}>1$. Furthermore, we have proven that the flow speed downstream of strong compressive fronts is subsonic. Therefore the $C_{ \pm}$: $d t / d x=1 /(u \pm c)$ and $C_{0}: d t / d x=1 / u$ characteristics assume the geometry depicted in Figure 6 for a stationary left-facing shock (there is no additional difficulty in considering nonstationary shocks). The front separates the $C_{-}$ characteristics, whereas the other two families simply cross it. The geometry of the characteristics for this case resembles that of compressive shocks in ordinary gas dynamics. Clearly, the $C_{-}$characteristic can also be traced back to the initial data, so the strong compression solutions satisfy the entropy condition. Hence the downstream state is determined uniquely from the upstream state.

2. For the case $S=I, p_{1}=p_{I}$, and the velocity relative to the mass-loading front is supersonic ahead $\left(u_{0}>c_{0}\right)$ and sonic behind $\left(u_{1}=c_{1}\right)$. Thus one of the downstream characteristics, in our case $C_{-}$, when viewed from downstream, corresponds to the mass-loading front. The characteristics are illustrated in Figure 7. Therefore the sonic condition downstream allows us to determine the downstream state from the upstream without further assumption.

3. The weak compression solutions $S$ to the R-H conditions lie on the weak compression branch for which $p_{I}>p_{1}$ $>p_{A}$. Since the momentum line always passes through the point $\left(\tau_{0} /(1-\bar{\alpha})^{2}, p_{0}\right)$ in the $(\tau, p)$ plane, we always have $p_{1}>p_{0}$. As we have noted, the gas flow on either side of the 
Rank and Oughton: Mass-Loading Shocks

9447

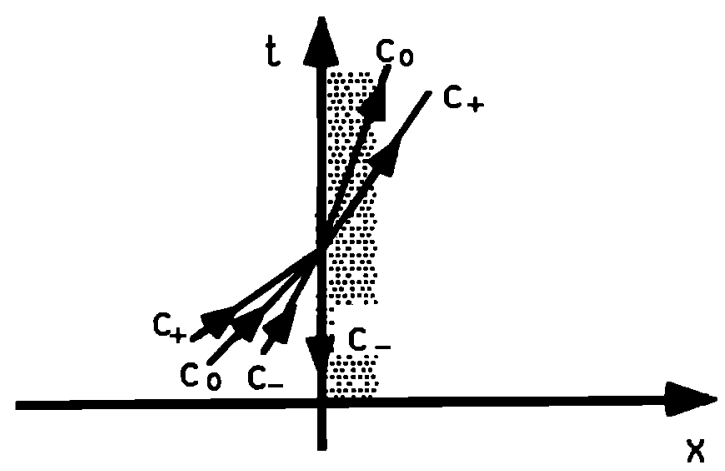

Fig. 7. Same as for Figure 6, except for downstream sonic case.

mass-loading front is supersonic, although mass loading does decelerate the incident flow. Each family of characteristics crosses the front, as illustrated in Figure 8. As discussed above, this implies that the system is nonconvex, and so $S$ and $\left(\tau_{0}, p_{0}\right)$ cannot be connected by a single wave front. In order to exclude discontinuities that move faster than the characteristics on both sides, it is necessary to use a compound wave to connect upstream and downstream states [Ole ni, 1965]. To construct the compound wave, connect $\left(\tau_{0}, p_{0}\right)$ first to the $S=I$ front and then follow by an isentropic centered rarefaction wave to reach the downstream state $S$. Such a compound wave is possible because the $S=I$ front moves at the downstream sound speed. If we adopt this compound wave to connect the states, then the downstream state is determined completely by the upstream state. If weak compression discontinuities were not exclued, then the solution of the initial value problem would not be unique.

4. In the case of weak expansion, $p_{0} \geq p_{1}>p_{I I}$, and the flow is subsonic on either side of the mass-loading front. As discussed, mass loading accelerates a subsonic flow so $u_{0}<$ $c_{0}, u_{1}<c_{1}$ and $u_{1}>u_{0}$. Consideration of the characteristics, illustrated in Figure 9, indicates that this is an indeterminate case. The solution for this case can presumably be determined uniquely only by considering a nonconstant mass-loading term and investigating the shock structure problem.

5. For $S$ on the strong expansion branch, $p_{1}<p_{I I}$, and the gas flow is subsonic ahead of the mass-loading front and supersonic behind. From Figure 10 it is evident that the front separates the $C_{\text {- }}$ characteristics, but this separation does not satisfy the geometrical entropy condition even though $S$

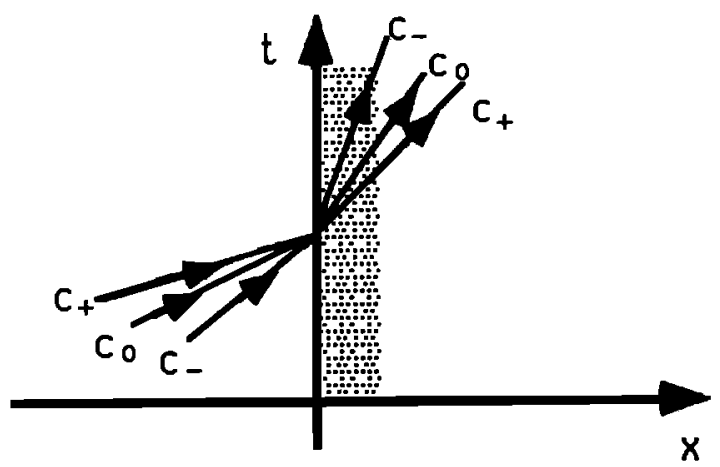

Fig. 8. Same as for Figure 6, except for weak compression.

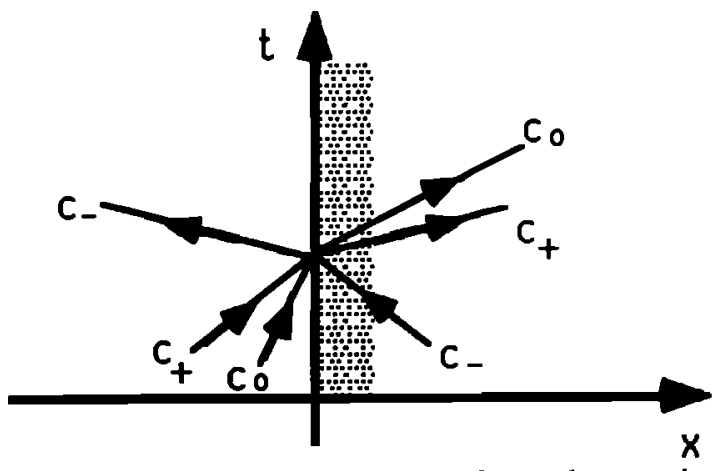

Fig. 9. Same as for Figure 6, except for weak expansion.

is consistent with the $\mathbf{R}-\mathrm{H}$ conditions. Accordingly, we exclude strong mass-loading expansions.

In conclusion, we have excluded weak mass-loading compressions and strong mass-loading expansions on the basis of purely geometrical entropy conditions. It is not at all clear how one might have arrived at this conclusion simply on the basis of thermodynamic entropy arguments (see Lank [1991] for further discussion on this point). Furthermore, we have established the existence of a compound wave consisting of an $S=I$ shock followed by an isentropic centered rarefaction wave which enables us to connect a supersonic upstream state to a supersonic downstream state provided $p_{I}>p_{1} \geq p_{A}$.

In closing this section, let us investigate the properties of mass-loading shocks as a function of the upstream Mach number. This has the added benefit of further clarifying the above analysis. The total energy relation (24) can be rewritten as

$\mu^{2} u_{0}^{2}+\left(1-\mu^{2}\right) c_{0}^{2}=(1+\bar{\alpha})\left(\mu^{2} u_{1}^{2}+\left(1-\mu^{2}\right) c_{1}^{2}\right)$,

for parallel shocks. If we denote by $c_{*}^{2}$ the common value of both sides, then

$$
\begin{aligned}
\left(1-\mu^{2}\right)\left(u_{0}^{2}-c_{0}^{2}\right) & =u_{0}^{2}-c_{*}^{2} ; \\
(1+\bar{\alpha})\left(1-\mu^{2}\right)\left(u_{1}^{2}-c_{1}^{2}\right) & =(1+\bar{\alpha}) u_{1}^{2}-c_{*}^{2},
\end{aligned}
$$

so that

$$
\begin{gathered}
\left|u_{0}\right|>c_{0} \Leftrightarrow\left|u_{0}\right|>c_{*} ; \\
\left|u_{1}\right|>c_{1} \Leftrightarrow\left|u_{1}\right|>c_{*} /(1+\bar{\alpha})^{1 / 2} .
\end{gathered}
$$

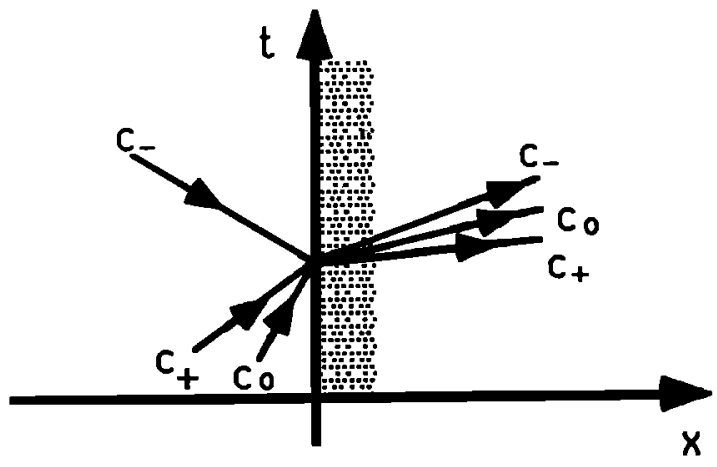

Fig. 10. Same as for Figure 6, except for strong expansion. 
Thus, because $\bar{\alpha} \neq 0, c_{*}$ no longer defines a critical sound speed as in the case of gas dynamics. By considering $\rho_{1} c_{*}^{2}$ and $\rho_{0} c_{*}^{2}$ together with the relation $\rho_{0} u_{0}^{2}+p_{0}=\rho_{1} u_{1}^{2}+$ $p_{1}$, we obtain

$$
c_{*}^{2}=\frac{p_{1}-p_{0}}{1 / \bar{\tau}_{1}-1 / \tau_{0}} .
$$

It follows then from (25) that

$$
\frac{u_{0} u_{1}}{c_{*}^{2}}=1+\frac{\bar{\alpha}}{1+\bar{\alpha}} \frac{M_{1}^{2}\left(\gamma M_{0}^{2}+1\right)}{M_{0}^{2}-M_{1}^{2}},
$$

which is the generalization of the well-known Prandtl relation

$$
u_{0} u_{1}=c_{*}^{2},
$$

for $\overline{\boldsymbol{\alpha}} \neq 0$. An expression relating the upstream Mach number to the downstream Mach number can be obtained either from (39) and (40) or directly from the quadratic (34).

$$
\begin{gathered}
\left(M_{1}^{2}-M_{0}^{2}\right)\left\{M_{1}^{2}\left[2 \gamma M_{0}^{2}-(\gamma-1)\right]-\left[2+(\gamma-1) M_{0}^{2}\right]\right\} \\
+\bar{\alpha}\left(2+(\gamma-1) M_{0}^{2}\right) M_{0}^{2}\left(1+\gamma M_{1}^{2}\right)^{2}=0 .
\end{gathered}
$$

Notice that the expression in the braces corresponds to the usual classical expression relating $M_{0}^{2}$ and $M_{1}^{2}$ for gas dynamics [e.g., Landau and Lifshitz, 1979, p. 331]. In deriving (41) we used the fact that the ratios of pressure and of density can be expressed in terms of the upstream and downstream Mach numbers as

$$
\begin{gathered}
\frac{p_{1}}{p_{0}}=\frac{1+\gamma M_{0}^{2}}{1+\gamma M_{1}^{2}} ; \\
\frac{\rho_{1}}{\rho_{0}}=(1+\bar{\alpha})^{2} \frac{1+1 / \gamma M_{1}^{2}}{1+1 / \gamma M_{0}^{2}} .
\end{gathered}
$$

Equation (41) can be solved numerically for some parameters of interest. In order to contrast the properties of gas dynamic shocks with those of mass-loading shocks, in Figure 11 we present plots of the square of the downstream Mach number, pressure, and density as functions of the square of the upstream Mach number for the case of ordinary gas dynamics. As usual, $M_{1}^{2}$ is hyperbolic in $M_{0}^{2}, p_{1} / p_{0}$ is linear in $M_{0}^{2}$, and $\rho_{1} / \rho_{0}$ tends asymptotically to $(\gamma+1) /(\gamma$ -1 ) $=4$ (for $\gamma=5 / 3$ ) as $M_{0}^{2} \rightarrow \infty$. The modifications introduced by a nonzero $\bar{\alpha}=\mathbf{0 . 0 1}$ are illustrated in Figure 12, and those for $\bar{\alpha}=0.001$ are illustrated in Figure 13. Branches $\mathrm{CB}$ and EF (B and $\mathrm{E}$ are the points at which $M_{1}^{2}=1$ ) resemble the upper and lower branches of the $M_{0}^{2}-M_{1}^{2}$ hyperbola for the $\bar{\alpha}=0$ case shown in Figure $11 a$. It is evident from Figure $12 a$ that, as we know already from the Hugoniot function, for a given $M_{0}^{2}$ there exist two possible downstream states (except at points $B$ and E). Also, as discussed above, not all the downstream states are physically realizable. On the basis of our analysis of the massloading Hugoniot, we can classify the $M_{0}^{2}-M_{1}^{2}$ massloading shock relation in a similar way. Points $\mathbf{B}$ and $\mathbf{E}$ correspond to sonic points I and II of the Hugoniot. Branch $\mathrm{AB}$ corresponds to accelerated subsonic-subsonic flows the existence of which we can neither prove nor disprove. Branch BC corresponds to strong expansion fronts, which

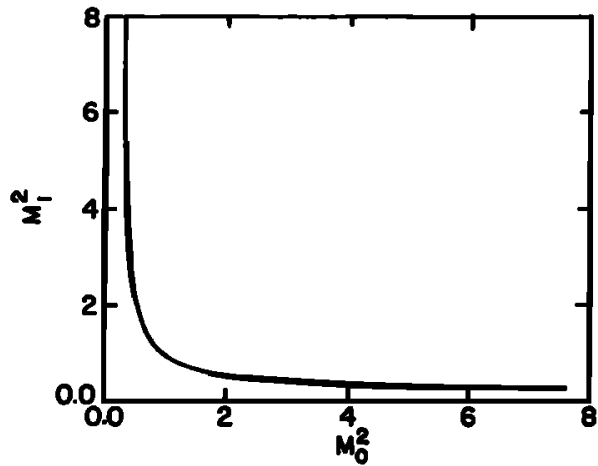

(b)
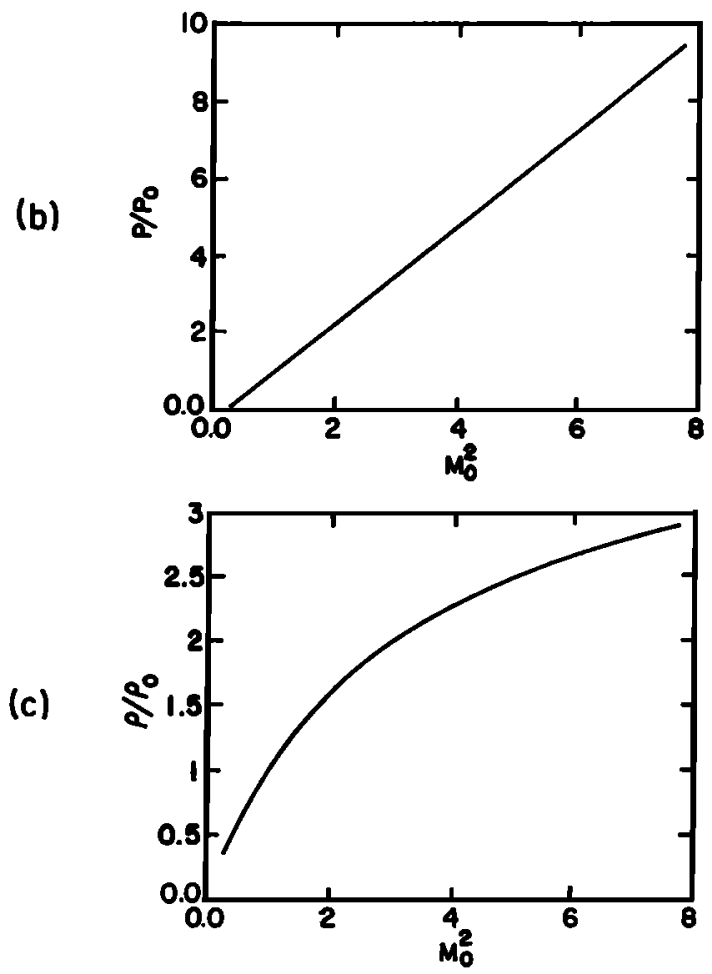

Fig. 11. Functions of the square of the incident Mach number for ordinary gas dynamics ( $\bar{\alpha}=0, \theta=0^{\circ}, \gamma=5 / 3$ ): (a) the square of the downstream flow Mach number, $(b)$ the downstream pressure, and $(c)$ the downstream density.

have been shown to be inadmissible solutions of the Rankine-Hugoniot conditions. The strong compression branch is $\mathrm{EF}$, and these represent unique, stable mass-loading shocks. The compound wave solutions corresponding to weak compressions lie on the locus DE. The corresponding behavior of the pressure and density ratios as a function of $M_{0}^{2}$ is easily seen on Figures $12 b$ and $12 c$. It is evident that the strong compression mass-loading shocks behave very like ordinary strong gas dynamic shocks.

\subsection{Oblique Shocks}

The results of section 3.1 are extended easily to oblique shocks for which $u_{y 0} \neq 0$. The main differences are a consequence of (24), since we now use $u^{2}=u_{x}^{2}+u_{y}^{2}$, as well as (23). Nevertheless, obliquity does introduce an important change in the Hugoniot function, which now becomes 
(a)

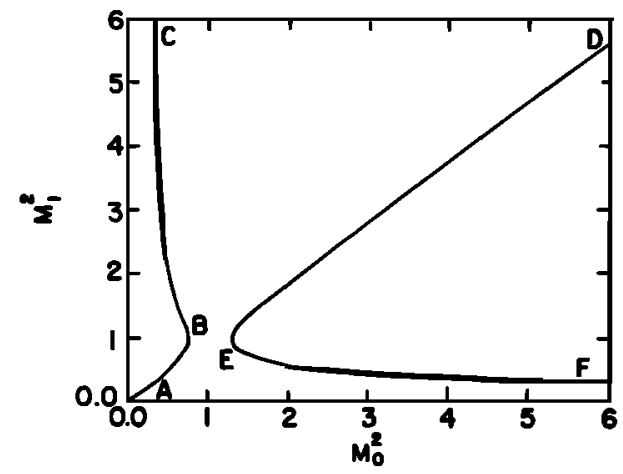

(b)

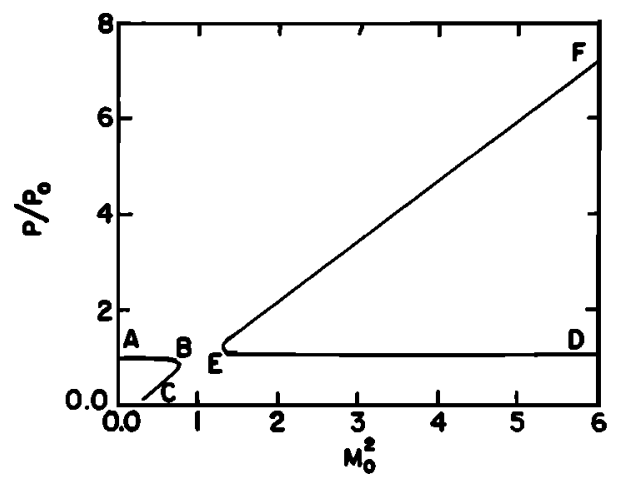

(c)

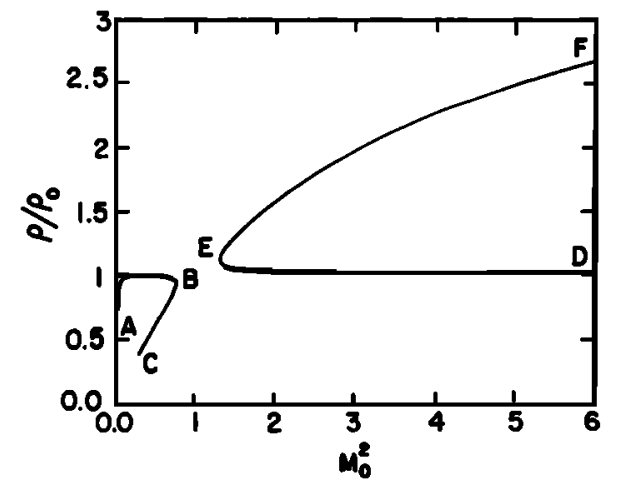

Fig. 12. Same as Figure 11, except mass loading is not neglected $\left(\bar{\alpha}=0.01, \theta=0^{\circ}, \gamma=5 / 3\right.$ ). See text for the interpretation of the different branches.

$$
\begin{array}{r}
H(\bar{\tau}, p)=\bar{\varepsilon}(\bar{\tau}, p)-\varepsilon\left(\tau_{0}, p_{0}\right)+\frac{p+p_{0}}{2}\left(\bar{\tau}-\tau_{0}\right) \\
-\frac{\bar{\alpha}}{2} m^{2} \tau_{0} \bar{\tau}-\frac{1}{2} \frac{\bar{\alpha}}{1+\bar{\alpha}} u_{y 0}^{2} .
\end{array}
$$

Equation (44) demonstrates that unlike the Hugoniot of ordinary gas dynamics, the mass-loading Hugoniot is not invariant with respect to tangential flows. While of interest here, its true significance is to be found in MHD with mass loading, and it is precisely this lack of invariance that induces the strong rotation of the magnetic field downstream of a mass-loading front such as a cometary bow shock. The lack of invariance is a consequence of the fact that the tangential velocity components in front of and behind the shock are unequal unless $\bar{\alpha}=0$, i.e.,

$$
u_{y 1}=\frac{1}{1+\bar{\alpha}} u_{y 0} .
$$

(a)

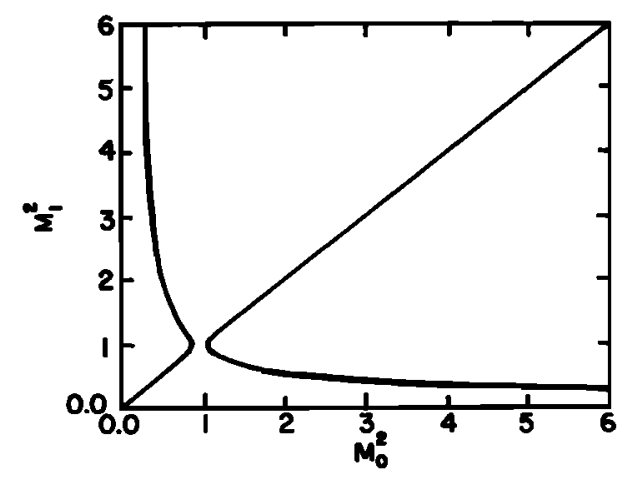

(b)

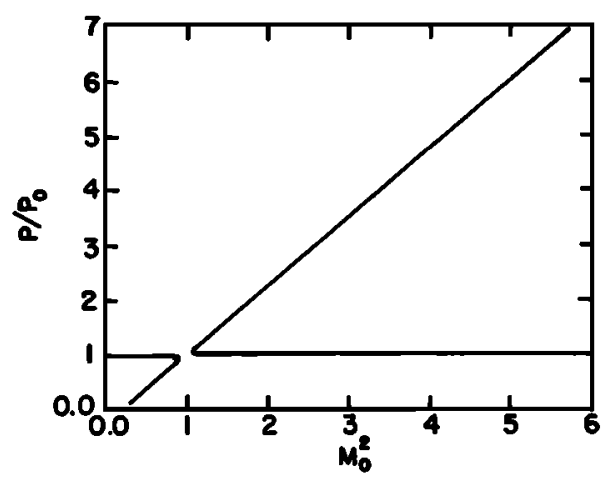

(c)

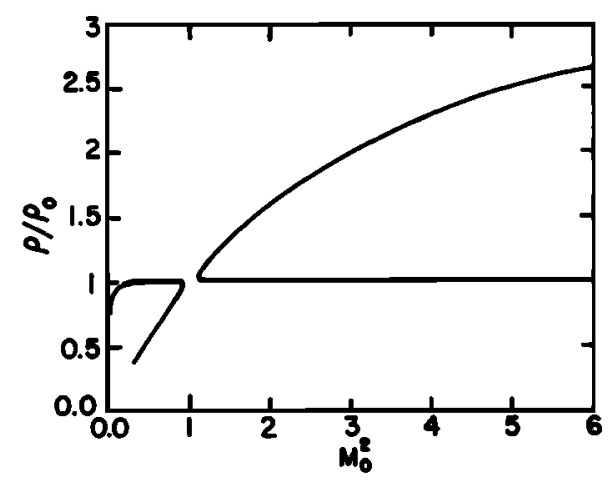

Fig. 13. Same as Figure 12 but with a reduced mass-loading rate $\left(\bar{\alpha}=0.001, \theta=0^{\circ}, \gamma=5 / 3\right)$. This parameter regime may be more appropriate to the inbound Halley bow shock.

This indicates that the bow shock is subjected to a shearing stress as a consequence of mass-loading, something that sets it apart both from ordinary nonreactive gas dynamical shocks and combustion shocks. We conjecture that such a shearing stress must lead eventually to the spontaneous destabilization of the shock front, presumably when the averaged mass-loading term $\bar{\alpha}$ exceeds some critical value. It would be of interest to explore this question further and, in particular, to determine the relationship of an instability criterion to the oblique tangency condition (50) below. For an ideal gas, (44) reduces to

$$
\begin{array}{r}
2 \mu^{2} H(\bar{\tau}, p)=\left(\bar{\tau}-\mu^{2} \tau_{0}\right) p-\left(\tau_{0}-\mu^{2} \bar{\tau}\right) p_{0}-\bar{\alpha} \mu^{2} m^{2} \tau_{0} \bar{\tau} \\
-\mu^{2} \frac{\bar{\alpha}}{1+\bar{\alpha}} m^{2} \tau_{0}^{2} \tan ^{2} \theta
\end{array}
$$

where $\theta$ is the angle the incident flow makes with the shock normal, so $u_{y 0}=u_{x 0} \tan \theta$. The Hugoniot relation can therefore be expressed as 


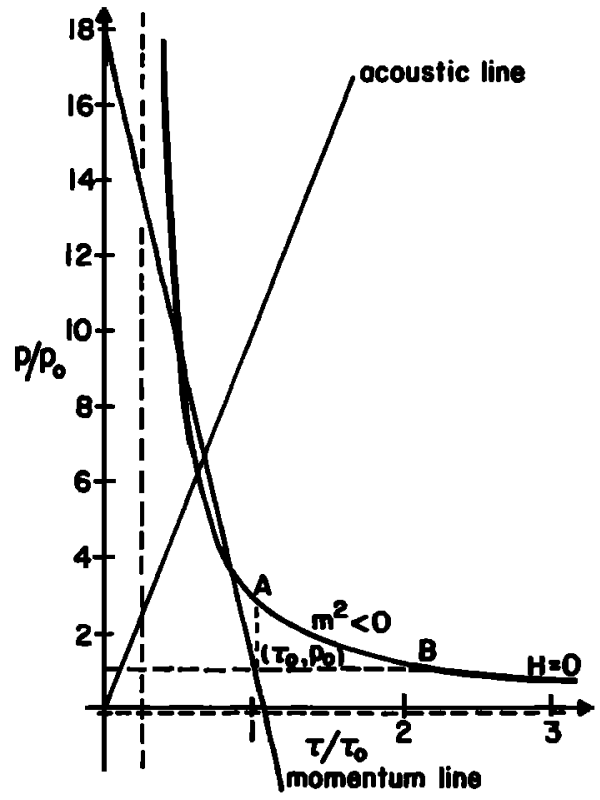

Fig. 14. Example of the mass-loading Hugoniot, together with graphical solutions of the R-H conditions, for oblique shocks $\left(M_{0}^{2}=\right.$ $10, \bar{\alpha}=0.01, \theta=80^{\circ}, \gamma=5 / 3$ ). Compare this figure with Figure $4 a$.

$$
\begin{aligned}
\frac{p}{p_{0}}=\frac{1-\mu^{2} \bar{\tau} / \tau_{0}}{\bar{\tau} / \tau_{0}-\mu^{2}}+ & \bar{\alpha} \mu^{2} \gamma M_{0}^{2} \frac{\bar{\tau} / \tau_{0}}{\bar{\tau} / \tau_{0}-\mu^{2}} \\
& +\mu^{2} \gamma M_{0}^{2} \frac{\bar{\alpha}}{1+\bar{\alpha}} \frac{\tan ^{2} \theta}{\bar{\tau} / \tau_{0}-\mu^{2}},
\end{aligned}
$$

where $M_{0}^{2}=u_{x 0}^{2} / \gamma p_{0} \tau_{0}$ is the square of the Mach number normal to the shock. The condition (30) which determines whether $\left(\tau_{0}, p_{0}\right)$ lies above or below the Hugoniot is relaxed somewhat, with the condition now given by

$$
M_{0}^{2}>\frac{1}{1+\bar{\alpha}} \frac{2}{\gamma-1} \frac{1}{1+\tan ^{2} \theta /(1+\bar{\alpha})^{2}} .
$$

The asymptotes of the Hugoniot remain unchanged. An example of the oblique Hugoniot, together with graphical solutions of the R-H conditions, is illustrated in Figure 14. Other than using $\theta=80^{\circ}$, all the remaining parameters are identical to those used in plotting Figure $4 a$. For the oblique Hugoniot a much larger branch is inadmissible (i.e., where $M_{0}^{2}<0$ ), and the strong compression fronts experience a comparatively smaller compression than their parallel counterparts, whereas the weak compression fronts are more strongly compressed.

As before, the $\mathrm{R}-\mathrm{H}$ conditions can be combined as a quadratic equation in $\bar{\tau} \equiv \bar{\tau} / \tau_{0}$,

$$
\begin{aligned}
& (1+\bar{\alpha}) \tilde{\tau}^{2}-\frac{2 \gamma}{\gamma+1}\left(1+\frac{1}{\gamma M_{0}^{2}}\right) \bar{\tau} \\
& \quad+\frac{\gamma-1}{\gamma+1}\left(1+\frac{\bar{\alpha}}{1+\bar{\alpha}} \tan ^{2} \theta\right)+\frac{2}{\gamma+1} \frac{1}{M_{0}^{2}}=0,
\end{aligned}
$$

with solutions

$$
\begin{aligned}
& \frac{\tau}{\tau_{0}}=\frac{\gamma}{\gamma+1} \frac{1}{(1+\bar{\alpha})^{2}}\left\{\left(1+\frac{1}{\gamma M_{0}^{2}}\right) \pm\left[\left(1+\frac{1}{\gamma M_{0}^{2}}\right)^{2}\right.\right. \\
& \left.\left.-(1+\bar{\alpha}) \frac{\gamma^{2}-1}{\gamma^{2}}\left(1+\frac{2}{\gamma-1} \frac{1}{M_{0}^{2}}+\frac{\bar{\alpha}}{1+\bar{\alpha}} \tan ^{2} \theta\right)\right]^{1 / 2}\right\}
\end{aligned}
$$

The tangential condition for the momentum line and the Hugoniot is now slightly more stringent in that we require

$$
(1+\bar{\alpha})=\frac{\gamma^{2}}{\gamma^{2}-1} \frac{\left(1+1 / \gamma M_{0}^{2}\right)^{2}}{1+\frac{2}{\gamma-1} 1 / M_{0}^{2}+\frac{\bar{\alpha}}{1+\bar{\alpha}} \tan ^{2} \theta}
$$

to hold. As for the case of parallel shocks, the points of intersection of the downstream sonic line, the normal momentum line, and the Hugoniot all coincide at the point of tangency defined by (50). Thus the analysis presented for parallel mass-loading fronts goes through unchanged for the case of oblique mass-loading fronts.

It is convenient to explore the properties of the $\mathrm{R}-\mathrm{H}$ solutions as a function of $M_{0}^{2}$, as was done earlier. To obtain an equation relating $M_{1}^{2}$ to $M_{0}^{2}$, one follows essentially the same procedure as outlined above to obtain

$$
\begin{gathered}
\left(M_{1}^{2}-M_{0}^{2}\right)\left\{M_{1}^{2}\left[2 \gamma M_{0}^{2}-(\gamma-1)\right]-\left[2+(\gamma-1) M_{0}^{2}\right]\right\} \\
+\bar{\alpha}\left(2+(\gamma-1) M_{0}^{2} \sec ^{2} \theta\right) M_{0}^{2}\left(1+\gamma M_{1}^{2}\right)^{2}=0 .
\end{gathered}
$$

Note that the "Prandtl relation" is yet more complicated than before and is given by

$$
\begin{aligned}
\frac{c_{*}^{2}}{u_{x 0} u_{x 1}}= & \frac{M_{0}^{2}-M_{1}^{2}}{M_{0}^{2}-M_{1}^{2}+\frac{\bar{\alpha}}{1+\bar{\alpha}} M_{1}^{2}\left(\gamma M_{0}^{2}+1\right)} \\
& +\mu^{2} \tan ^{2} \theta \frac{M_{0}^{2}\left(\gamma M_{1}^{2}+1\right)}{M_{1}^{2}\left(\gamma M_{0}^{2}+1\right)} \\
& \cdot \frac{M_{0}^{2}-M_{1}^{2}}{M_{0}^{2}\left(\gamma M_{1}^{2}+1\right)-\frac{1}{1+\bar{\alpha}} M_{1}^{2}\left(\gamma M_{0}^{2}+1\right)}
\end{aligned}
$$

The relations (42) and (43) hold without change.

Numerical solutions of (51), (42), and (43) are presented in Figure 15 (for $\theta=30^{\circ}$ ) and 16 (for $\theta=80^{\circ}$ ). The interpretation of these figures is substantially the same as that for the parallel mass-loading front case, and the differences in properties are readily perceived.

\section{Conclusions}

Mass loading is ubiquitous, occurring throughout the solar system. It is of importance both at the large-scale (magneto)hydrodynamic level as well as at the microphysical. With the in situ exploration of comets Halley and G-Z, as well as of Venus and Mars, we now have a unique opportunity to investigate the physics and properties of shocks subjected to mass loading. In this paper we have considered the simplest possible hydrodynamical model for both parallel and oblique shocks with a view to laying the foundations for future 
(a)

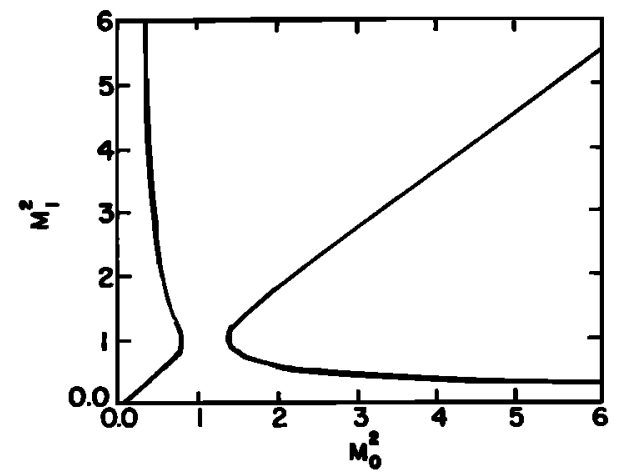

(b)

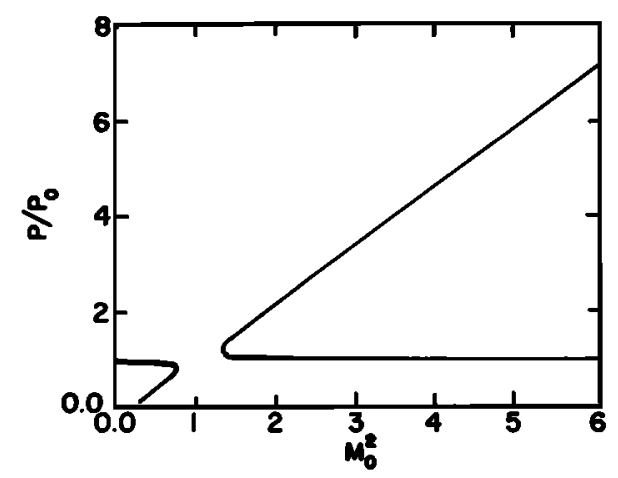

(c)

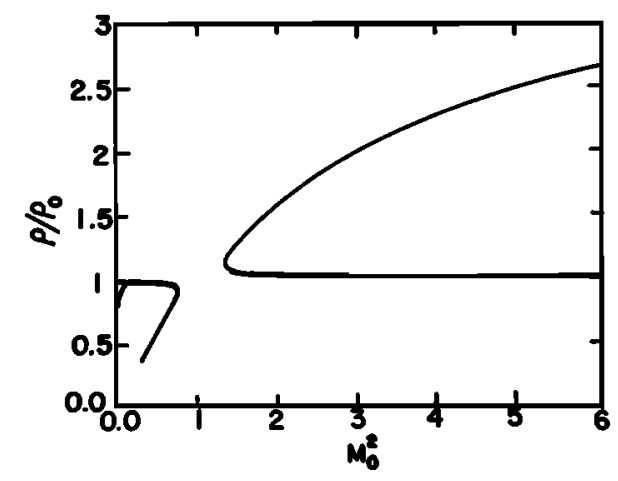

Fig. 15. Functions of square of incident Mach number for oblique mass-loading shocks $\left(\bar{\alpha}=0.01, \theta=30^{\circ}, \gamma=5 / 3\right)$ : $(a)$ square of downstream flow Mach number, $(b)$ downstream pressure, and (c) downstream density.

extensions to MHD. To ascertain which of the possible downstream states represent a physically acceptable solution to the boundary conditions, we found it necessary to abandon familiar thermodynamical arguments in favor of a geometrical entropy condition. The geometrical entropy condition is a consequence of requiring that a shock be determined uniquely from its initial data and that, for ordinary gas dynamics, both the thermodynamical and the geometrical entropy conditions coincide. It turns out that the entropy condition is surprisingly simple to use and admits a very nice geometrical interpretation.

As usual, the properties of mass-loading gas dynamic shocks can all be understood in terms of the Hugoniot function. From the Hugoniot we have established that the gas dynamics of mass-loading flows resembles more closely that of gas dynamics with combustion than of nonreacting gas dynamics. Thus the Hugoniot has been shown to possess two distinguished points, which correspond to the ChapmanJouguet points on the combustion Hugoniot. These distinguished points (points I and II of Figure 5) separate super- (a)

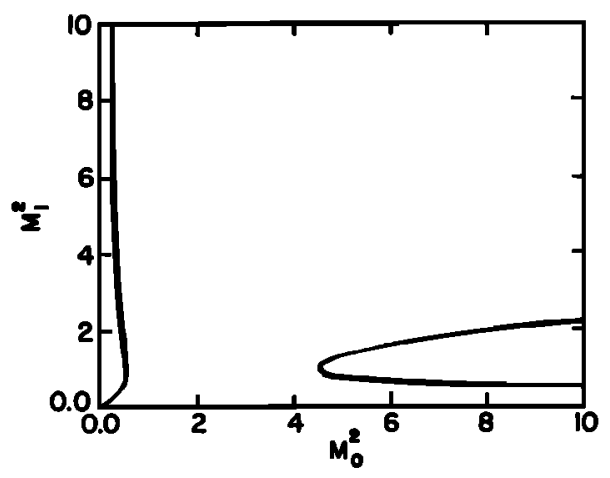

(b)

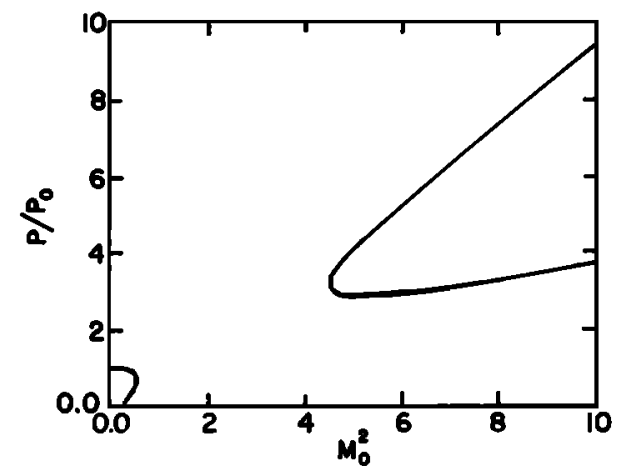

(c)

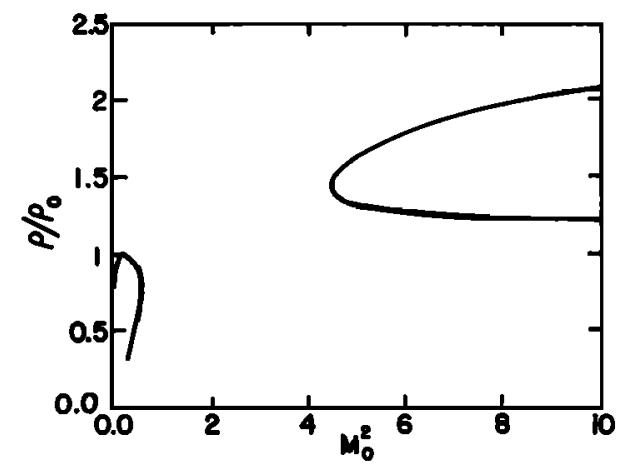

Fig. 16. The same as in Figure 14, except with $\theta=80^{\circ}$.

sonic from subsonic downstream states. It is interesting that points I and II correspond to turning point solutions of an equation which has close similarities to one derived first by Biermann et al. [1967] to show that a shock must form eventually in a sufficiently strongly accreting flow. However, as we have discussed, both equations have quite different interpretations, BBS having considered effectively a shock structure problem, whereas we consider instead the effects of averaged, steady mass injection at the shock.

What we have called the strong compression shock is fairly similar to an ordinary gas dynamic shock in that the flow velocity across the mass-loading front is decelerated from supersonic to subsonic velocities. Nevertheless, there are differences, one being that the upstream flow can never be sonic but instead must exceed some critical downstream Mach number $M_{\text {crit }}>1$, defined by the tangency condition for the Hugoniot. Furthermore, there is no gas dynamical analog of the weakly compressive mass-loading shock for which the incident supersonic flow velocity, although decelerated, nevertheless remains supersonic downstream. We have shown that as a consequence of the geometrical entropy condition, a weakly compressive mass-loading front has a compound wave structure. The flow velocity profile of 
such a shock is that of an initially uniform supersonic velocity with a sharp dip at which the flow velocity drops to $u_{x}=c$, followed by an increasing velocity which tends asymptotically from below to $u_{x}=u_{x 1}>c_{1}, u_{x 1}<u_{x 0}$. Qualitatively similar structures have been observed at the inbound Halley bow shock, with Coates et al. [1987b, p. 60] reporting that "the [shock] structure is complicated with a short-lived decrease preceding a more permanent decrease in speed." The same data are illustrated again by Coates et al. [1990, Figure 3] (between the labels $S_{1}$ and $S_{2}$ ), and the shock profile is quite dramatic. The solar wind speed drops off from about $300 \mathrm{~km} \mathrm{~s}^{-1}$ to less than $200 \mathrm{~km} \mathrm{~s}^{-1}$ (their point A) before recovering to achieve a speed of $\sim 275 \mathrm{~km}$ $\mathrm{s}^{-1}$. The solar wind flow maintains this speed for a short distance (although greater than the thickness of the initial deceleration) before dropping off to almost the identical downstream speed of $200 \mathrm{~km} \mathrm{~s}^{-1}$ (their point B) as before. Again, the fluid recovers, but this time to achieve a speed of only $230 \mathrm{~km} \mathrm{~s}^{-1}$. Indeed, continuous mass loading downstream of a compound shock must eventually induce the still supersonic downstream flow to undergo a further shock transition. Whether such observed features can be explained in terms of successive compound mass-loading fronts needs to be resolved both numerically and by more detailed observational tests. For example, do the points labeled A and $B$ by Coates et al. [1990] on Figure 3 correspond to downstream sonic points at which $u=c$, and are the subsequent recovery speeds in excess of the local sound speed? Unfortunately, the observations may be complicated somewhat by the presence of a magnetic field. Nevertheless, it may be of interest to recast the data in terms of the variables used in section 3 in order to use the results developed here to classify and clarify the observed shock structures. Finally, we have discussed the shock properties in terms of the incident Mach number, and this reveals, given the shock Mach numbers cited by Coates et al. [1990], that supersonic-supersonic transitions should not be discounted.

The properties of the oblique incident flow case are readily understood and interpreted in much the same way as are parallel shocks. An important difference, however, is that mass loading subjects an oblique shock to shearing stresses. This certainly has important consequences for magnetohydrodynamic shocks (as will be discussed elsewhere), and we have conjectured that such shearing stresses may lead, under some circumstances, to unstable shocks. This remains to be investigated.

\section{APPENDIX}

If $\bar{\alpha}$ is regarded as a small parameter, then linearization of the oblique Rankine-Hugoniot conditions (21)-(24) yields

$$
\begin{gathered}
\frac{\delta \rho}{\rho_{0}}=\bar{\alpha}-\frac{\delta u_{x}}{u_{x 0}} \\
\frac{\delta p}{\rho_{0} u_{x 0}^{2}}=-\bar{\alpha}-\frac{\delta u_{x}}{u_{x 0}} \\
\frac{\delta u_{y}}{u_{y 0}}=-\bar{\alpha} ;
\end{gathered}
$$

$$
u_{x 0} \delta u_{x}+u_{y 0} \delta u_{y}+\delta w=-\bar{\alpha}\left(\frac{1}{2} u_{0}^{2}+w_{0}\right)
$$

where $w=\gamma /(\gamma-1) p / \rho$ and

$$
\delta w=\frac{\gamma}{\gamma-1}\left(\frac{\delta p}{\rho_{0}}-\frac{p_{0}}{\rho_{0}^{2}} \delta \rho\right) .
$$

It then follows that

$$
\frac{\delta u_{x}}{u_{x 0}}=\frac{\gamma+1}{2} \bar{\alpha} \frac{M_{0}^{2}}{1-M_{0}^{2}},
$$

from which we see that

$$
\begin{aligned}
& M_{0}^{2}>1 \Rightarrow \delta u_{x}<0 ; \\
& M_{0}^{2}<1 \Rightarrow \delta u_{x}>0 .
\end{aligned}
$$

Acknowledgments. We are particularly grateful to N. F. Ness, W. H. Matthaeus, F. Neubauer, G. M. Webb, J. F. McKenzie, and W. I. Axford for many stimulating conversations and suggestions related to this work. We are grateful also to Alex Fullerton for his help with the graphics. This work has been supported in part by the Bartol Space Physics Theory Program. Finally, we thank the referees for their very constructive suggestions.

The Editor thanks G. M. Webb and F. M. Neubauer for their assistance in evaluating this paper.

\section{REFERENCES}

Axford, W. I., The interaction of solar wind with comets, Planet. Space Sci., 12, 719, 1964.

Axford, W. I., E. Leer, and J. F. McKenzie, The structure of cosmic ray shocks, Astron. Astrophys., 111, 317, 1982.

Bame, S. J., et al., Comet Giacobini-Zinner: Plasma description, Science, 232, 356, 1986.

Biermann, L., B. Brosowski, and H. U. Schmidt, The interaction of the solar wind with a comet, Sol. Phys., 1, 254, 1967.

Coates, A., et al., Giotto measurements of cometary and solar wind plasma at the comet Halley bow shock, Nature, 327, 489, $1987 a$.

Coates, A. J., A. D. Johnstone, M. F. Thomsen, V. Formisano, E. Amata, B. Wilken, K. Jockers, J. D. Winningham, H. Borg, and D. A. Bryant, Solar wind flow through the comet Halley bow shock, Astron. Astrophys., 187, 55, $1987 b$.

Coates, A. J., A. D. Johnstone, R. L. Kessel, D. E. Huddleston, B. Wilken, K. Jockens, and F. M. Neubauer, Plasma parameters near the comet Halley bow shock, J. Geophys. Res., 95, 20,701, 1990.

Galeev, A. A., et al., The position and structure of the comet Halley bow shock: VEGA-1 and VEGA-2 measurements, Geophys. Res. Lett., 13, 841, 1986.

Ip, W.-H., and W. I. Axford, The plasma, in Physics and Chemistry of Comets, edited by W. F. Huebner, pp. 177-232, SpringerVerlag, New York, 1989.

John, F., Partial Differential Equations, Springer-Verlag, New York, 1982.

Krankowsky, D., et al., In situ gas and ion measurements at comet Halley, Nature, 321, 347, 1986.

Landau, L. D., and E. M. Lifshitz, Fluid Mechanics, Pergamon, Elmsford, N. Y., 1979.

Lax, P. D., Hyperbolic systems of conservation laws and the mathematical theory of shock waves, Reg. Conf. Ser. Appl. Math., Soc. Ind. Appl. Math., Philadelphia, Pa., 1973.

Liberman, M. A., and A. L. Velikovich, Physics of Shock Waves in Gases and Plasmas, Springer-Verlag, New York, 1986.

Mukai, T., W. Miyake, T. Terasawa, M. Kitayama, and K. Hirao, Plasma observations by Suisei of the solar wind interaction with the comet Halley, Nature, 321, 299, 1986.

Neubauer, F. M., et al., First results from the Giotto magnetometer experiment at comet Halley, Nature, 321, 352, 1986.

Neubauer, F. M., K. H. Glassmeier, M. H. Acuna, F. Mariani, N. F. Ness, and A. J. Coates, Giotto magnetic field observations at 
the outbound quasi-parallel bow shock of comet Halley, Ann. Geophys., 8, 463, 1990.

Neugebauer, M., et al., The pick-up of cometary protons by the solar wind, Astron. Astrophys., 187, 21, 1987.

Oleǐnik, O. A., Uniqueness and stability of the generalized solution of the Cauchy problem for a quasi-linear equation, Usp. Mat. Nauk, 14, 165, 1959. (Am. Math. Soc. Transl. Ser. 2, Engl. Transl., 285, 33, 1965.)

Omidi, N., and D. Winske, A kinetic study of solar wind mass loading and cometary bow shocks, J. Geophys. Res., 92, 13,409, 1987.

Sauer, K., A new plasma model for comet Halley, Preprint 1/88, Akad. der Wiss. der DDR, Inst. für Kosmosforsch., Berlin, 1988. Smith, E. J., T. T. Bruce, J. A. Slavin, D. E. Jones, G. L. Siscoe, and D. A. Mendis, International Cometary Explorer encounter with Giacobini/Zinner: Magnetic field observations, Science, 232, $382,1986$.
Wallis, M. K., Shock-free deceleration of the solar wind?, Nature, 233, 23, 1971.

Wallis, M. K., Weakly-shocked flows of the solar wind plasma through atmospheres of comets and planets, Planet. Space Sci., $21,1647,1973$.

Weyl, H., Shock waves in arbitrary fluids, Commun. Pure Appl. Math., 2, 103, 1949.

Zank, G. P., Structure of the Halley bow shock, Planet. Space Sci., $38,1355,1990$.

Zank, G. P., Weyl's theorem for MHD, J. Plasma Phys., in press, 1991.

S. Oughton and G. P. Zank, Bartol Research Institute, The University of Delaware, Newark, DE 19716.

(Received November 16, 1990; revised February 21, 1991; accepted February 26, 1991.) 